Supporting Information

\title{
Two-Step Transformation of Aliphatic Polyketones into $\pi$-Conjugated Polyimines
}

Yumehiro Manabe, ${ }^{\dagger}$ Mitsuharu Uesaka, ${ }^{\dagger}$ Tomoki Yoneda, ${ }^{\dagger}$ and Yasuhide Inokuma ${ }^{* \dagger+}$

${ }^{\dagger}$ Division of Applied Chemistry, Faculty of Engineering, Hokkaido University, Kita 13, Nishi 8 Kita-ku, Sapporo, Hokkaido 060-8628, Japan

*Institute for Chemical Reaction Design and Discovery (WPI-ICReDD), Hokkaido University, Kita 21, Nishi 10, Kita-ku, Sapporo, Hokkaido 001-0021, Japan

\section{Table of contents}

I. NMR Spectra S-1

II. X-ray Structure $\quad$ S-7

III. DFT Calculations $\quad$ S-8

IV. Cadmium complex $\quad$ S-11

V. Synthesis of Polymer Materials $\quad$ S-12

VI. Characterization of Polymer Materials $\quad$ S-14

VII. Cartesian coordinates of optimized structures S-19 
I. NMR spectra

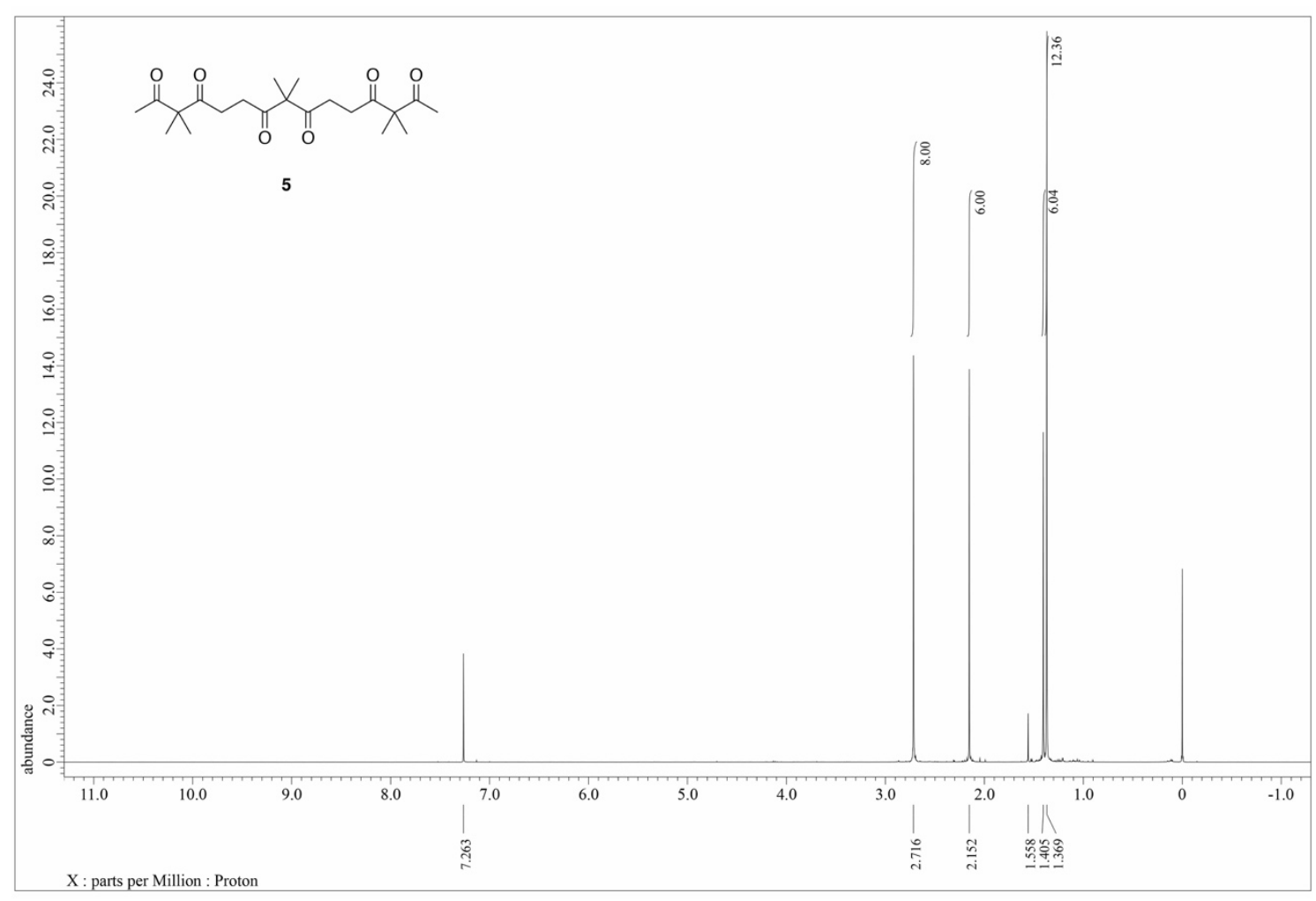

Figure S1. ${ }^{1} \mathrm{H}$ NMR spectrum of compound 5 in $\mathrm{CDCl}_{3}$.

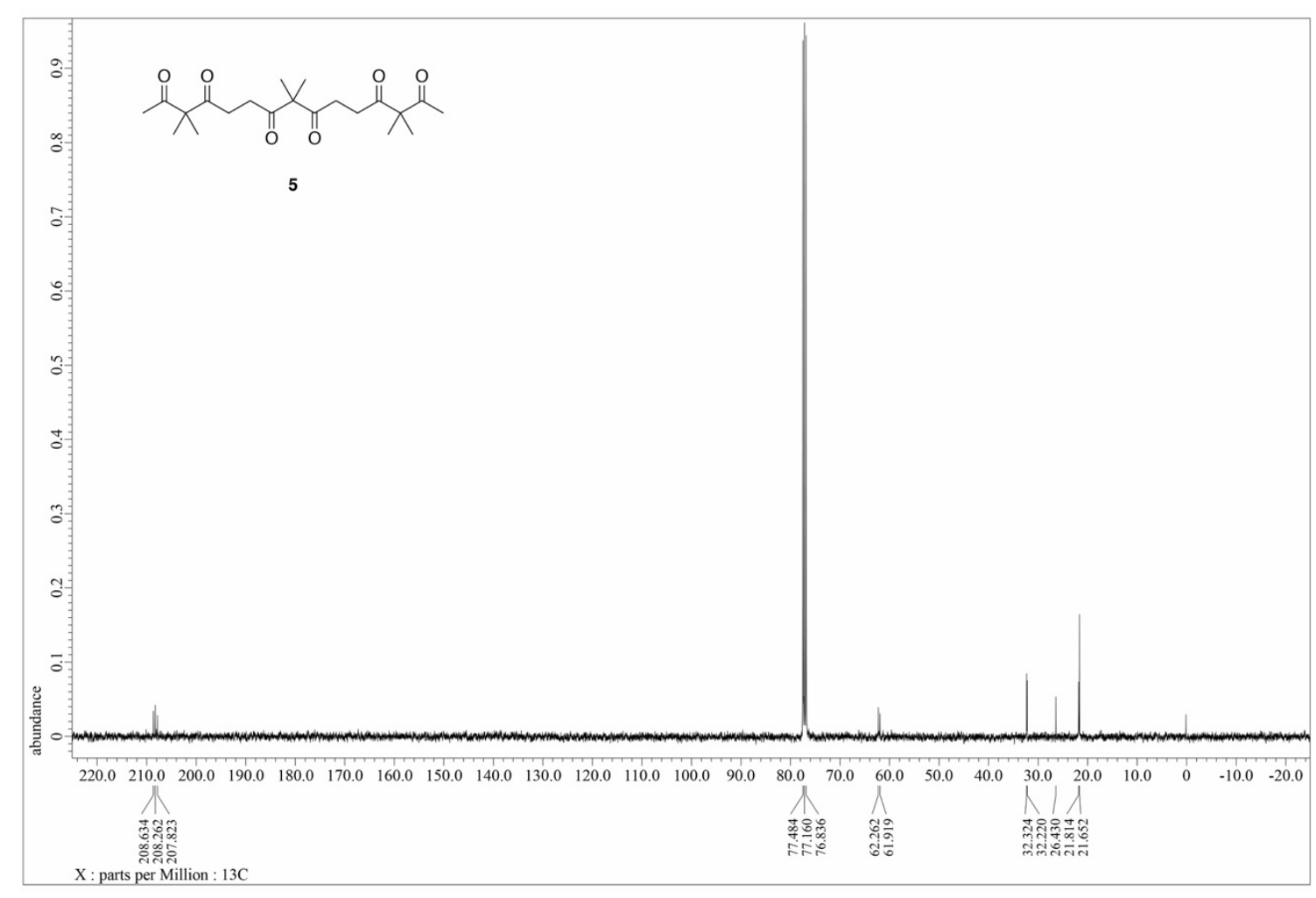

Figure S2. ${ }^{13} \mathrm{C}\left\{{ }^{1} \mathrm{H}\right\}$ NMR spectrum of compound $\mathbf{5}$ in $\mathrm{CDCl}_{3}$.

S-1 


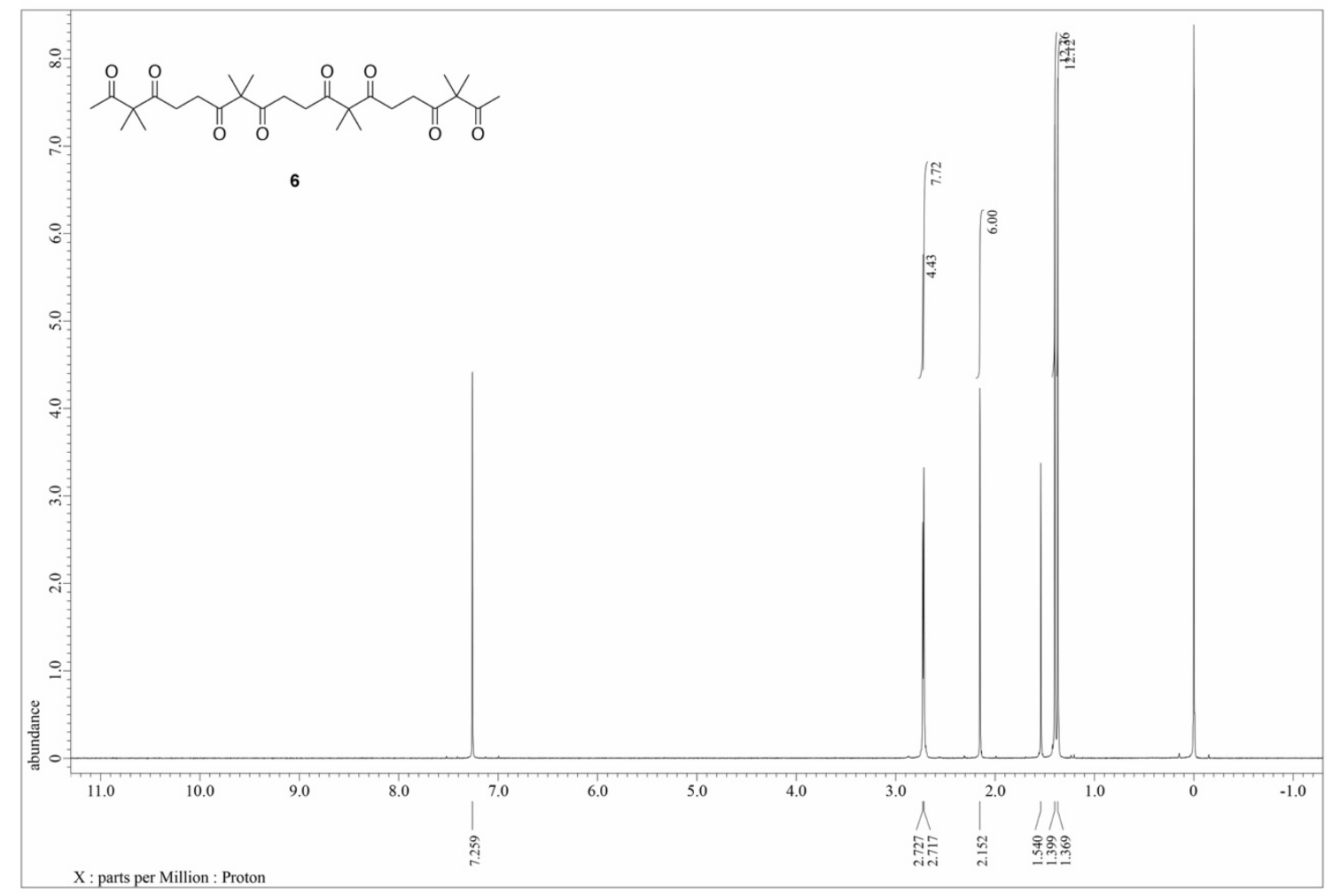

Figure S3. ${ }^{1} \mathrm{H}$ NMR spectrum of compound 6 in $\mathrm{CDCl}_{3}$ for the confirmation of the purity of starting material. Note that original spectra and characterization data have been already reported in reference 4. 


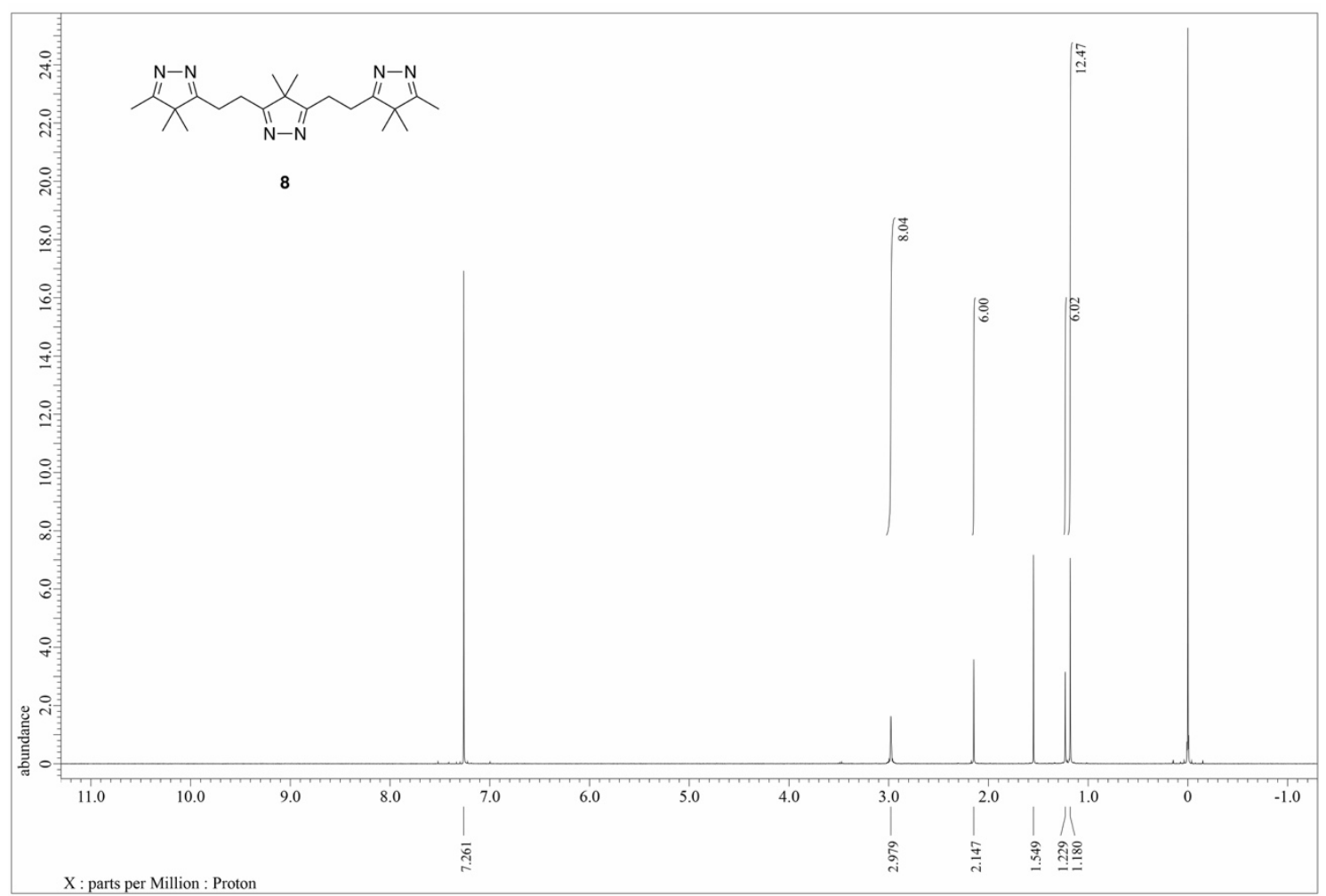

Figure S4. ${ }^{1} \mathrm{H}$ NMR spectrum of compound $\mathbf{8}$ in $\mathrm{CDCl}_{3}$.

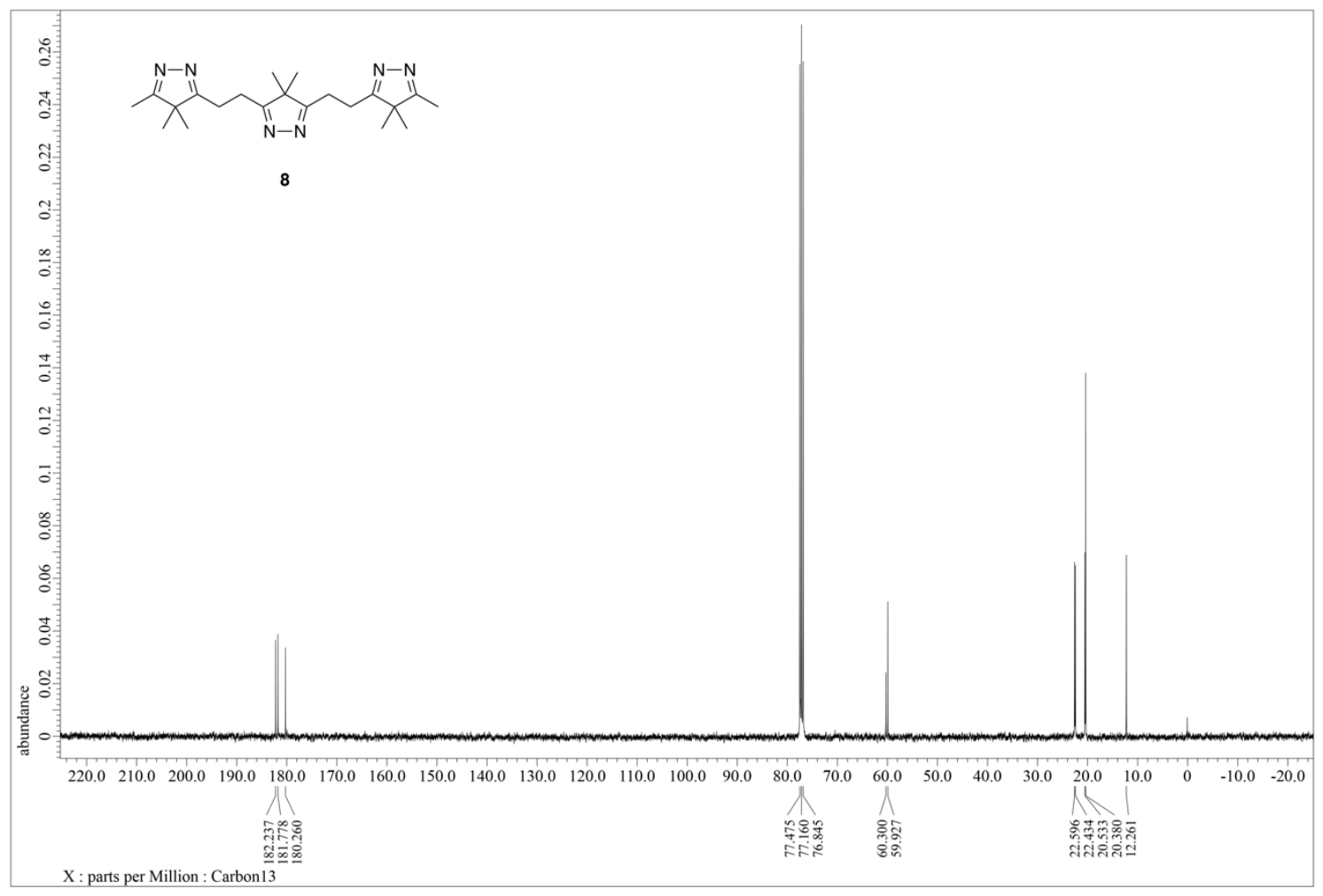

Figure S5. ${ }^{13} \mathrm{C}\left\{{ }^{1} \mathrm{H}\right\}$ NMR spectrum of compound $\mathbf{8}$ in $\mathrm{CDCl}_{3}$. 


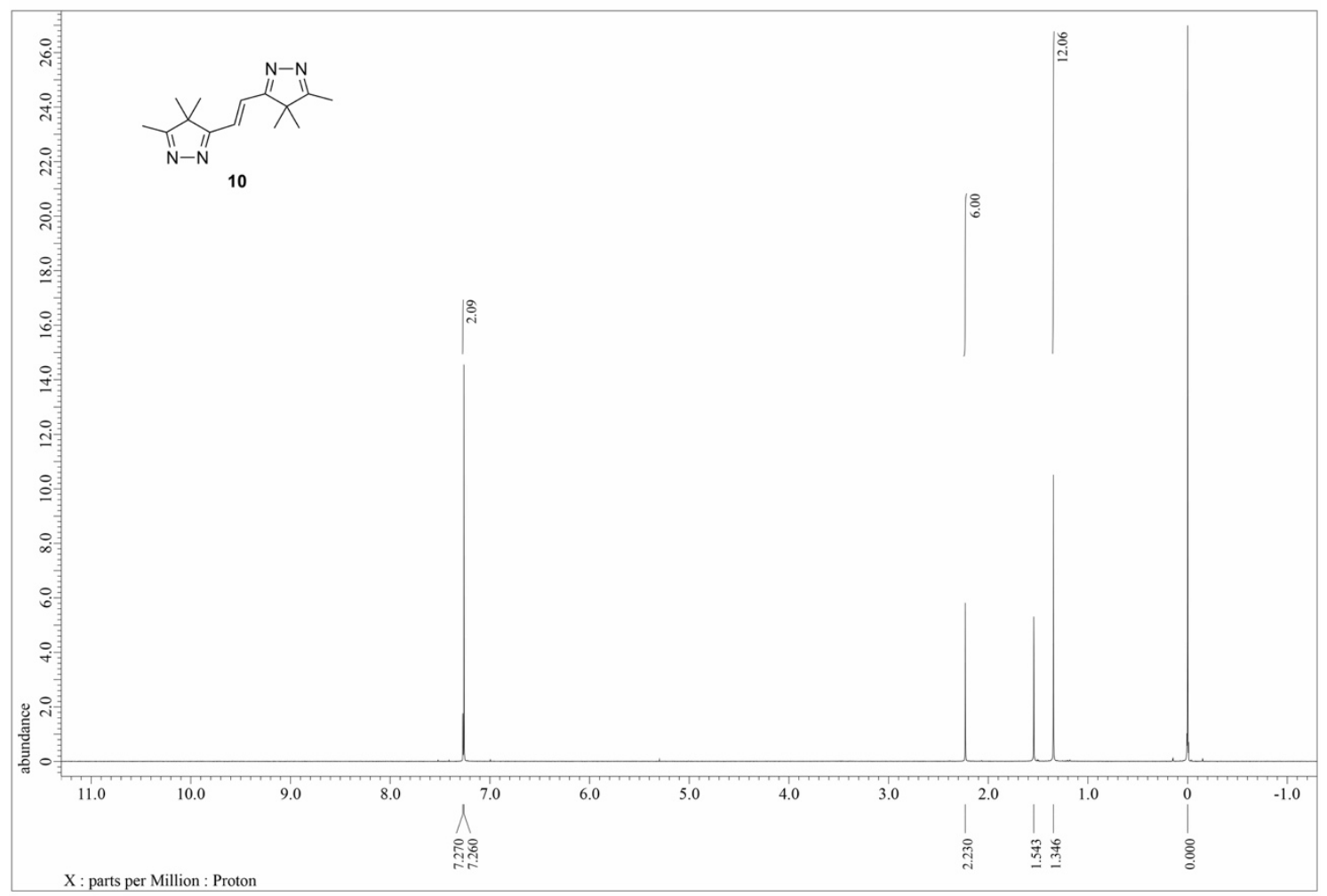

Figure S6. ${ }^{1} \mathrm{H}$ NMR spectrum of compound 10 in $\mathrm{CDCl}_{3}$.

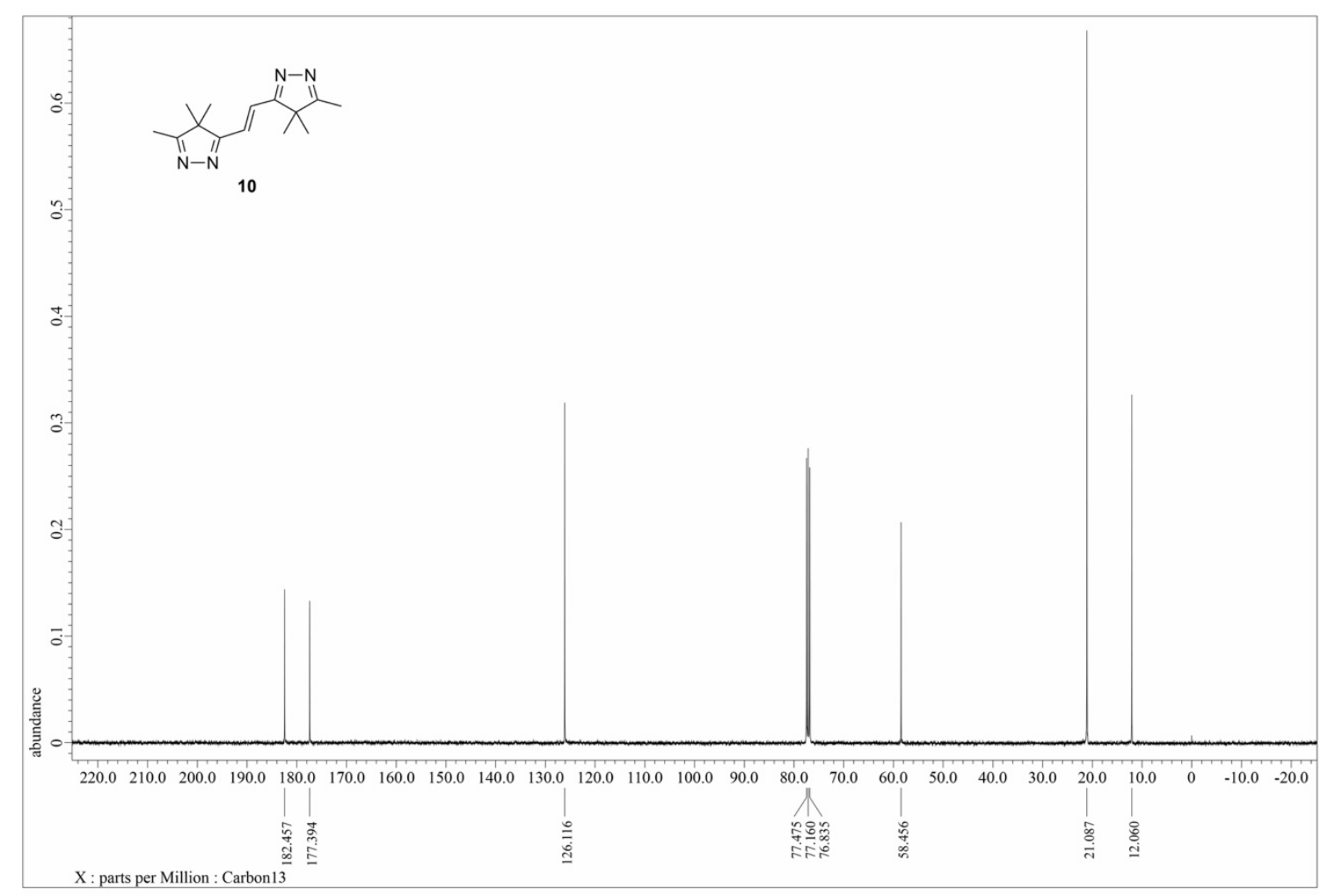

Figure S7. ${ }^{13} \mathrm{C}\left\{{ }^{1} \mathrm{H}\right\}$ NMR spectrum of compound $\mathbf{1 0}$ in $\mathrm{CDCl}_{3}$. 


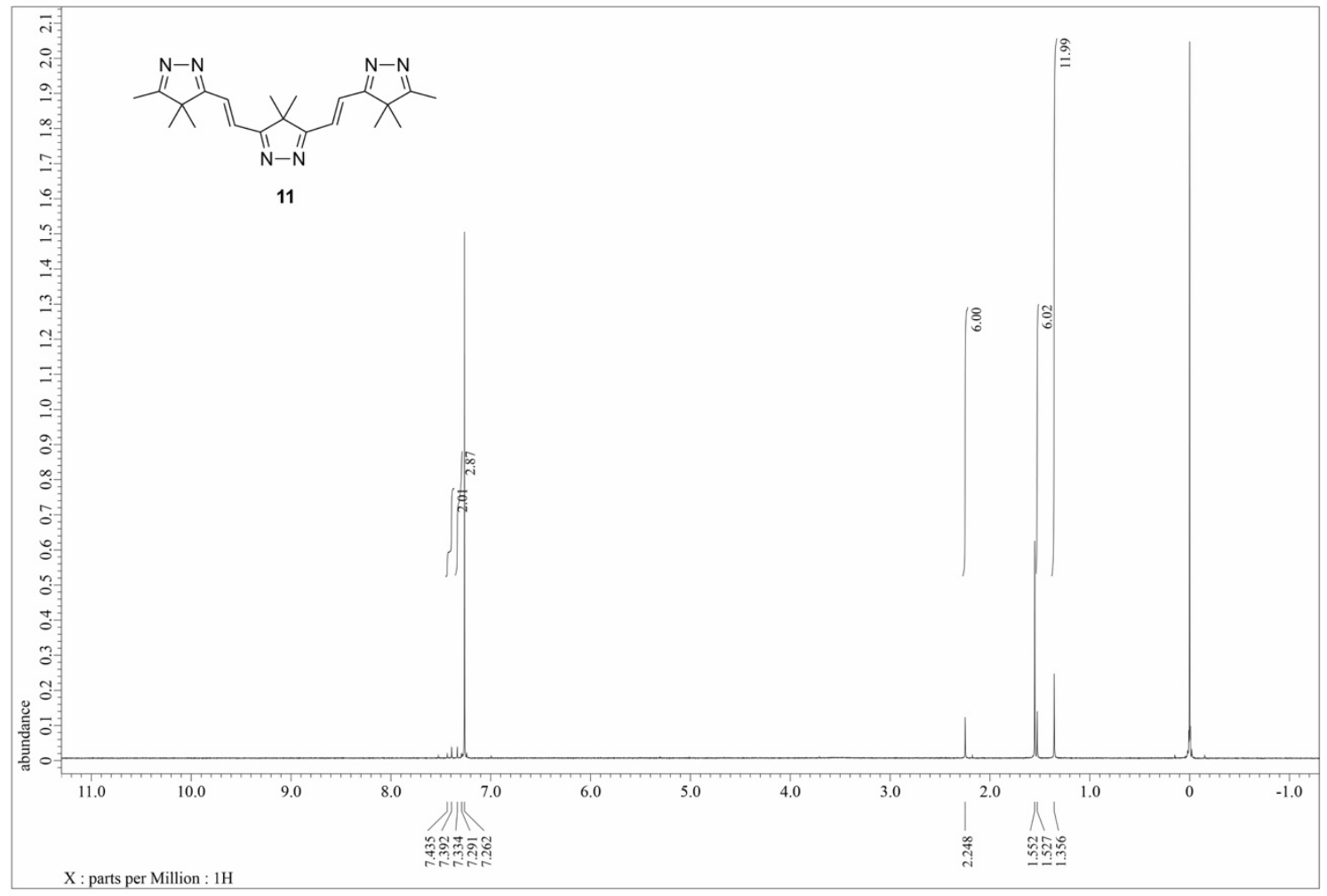

Figure S8. ${ }^{1} \mathrm{H}$ NMR spectrum of compound 11 in $\mathrm{CDCl}_{3}$.

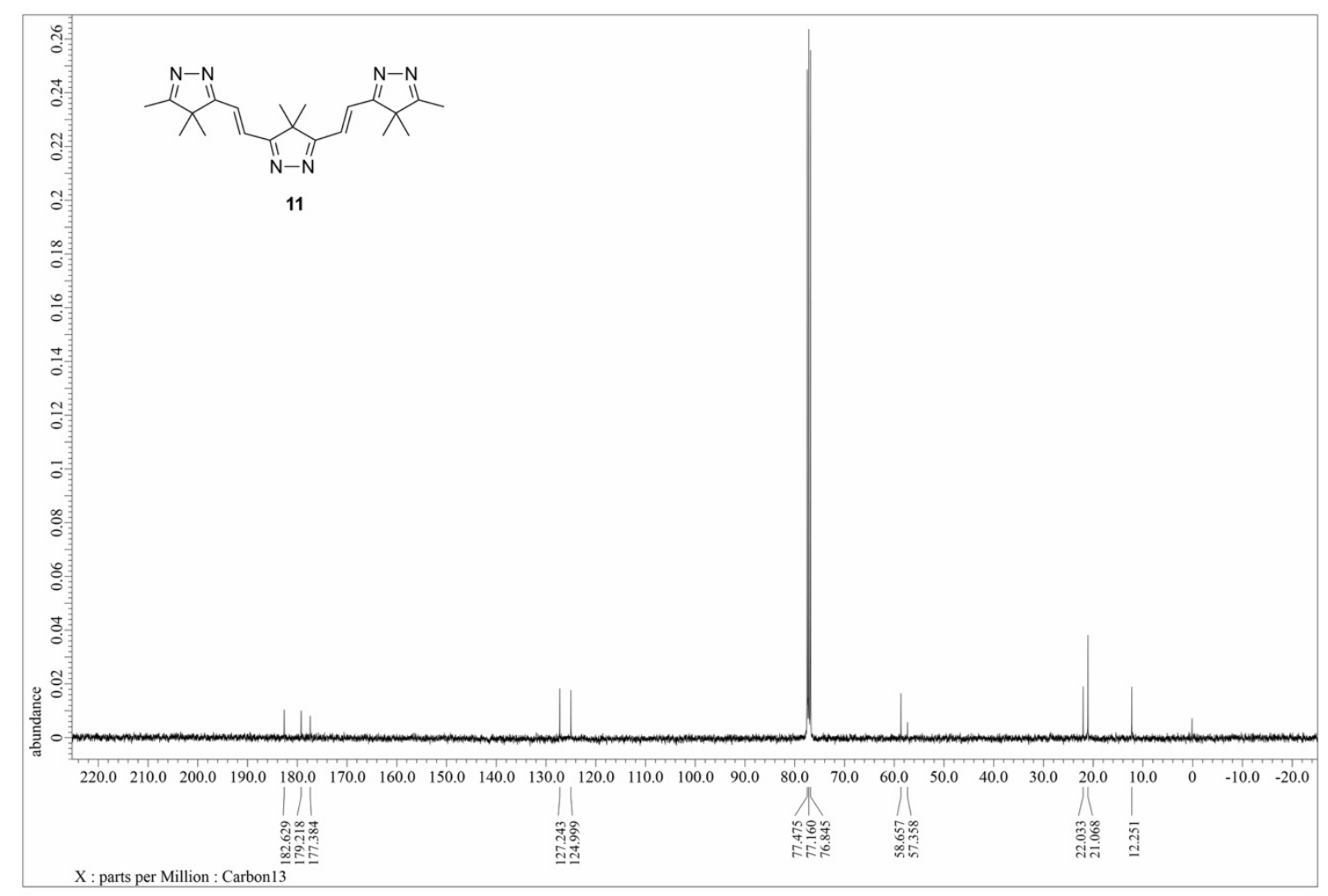

Figure S9. ${ }^{13} \mathrm{C}\left\{{ }^{1} \mathrm{H}\right\}$ NMR spectrum of compound 11 in $\mathrm{CDCl}_{3}$. 


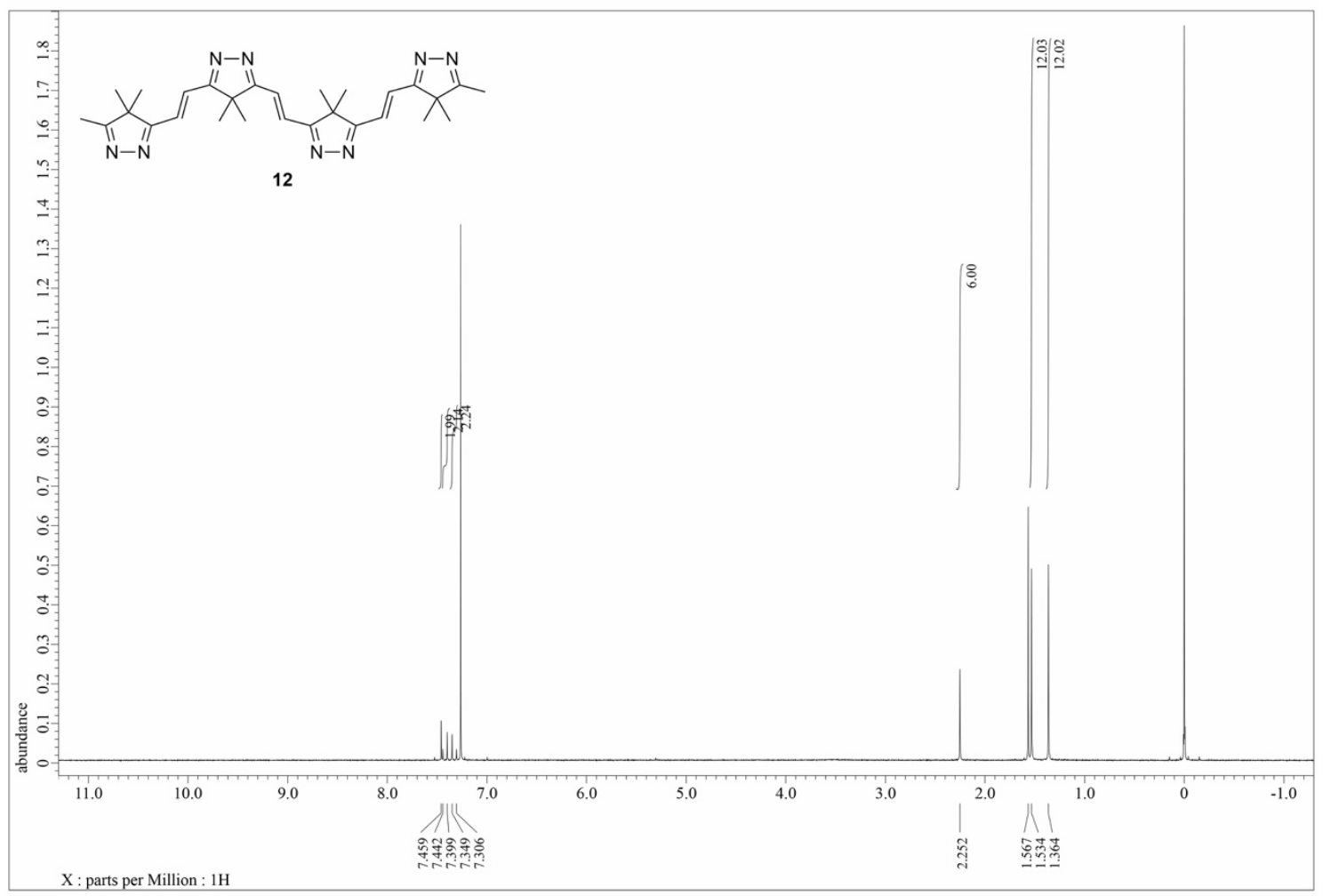

Figure S10. ${ }^{1} \mathrm{H}$ NMR spectrum of compound 12 in $\mathrm{CDCl}_{3}$.

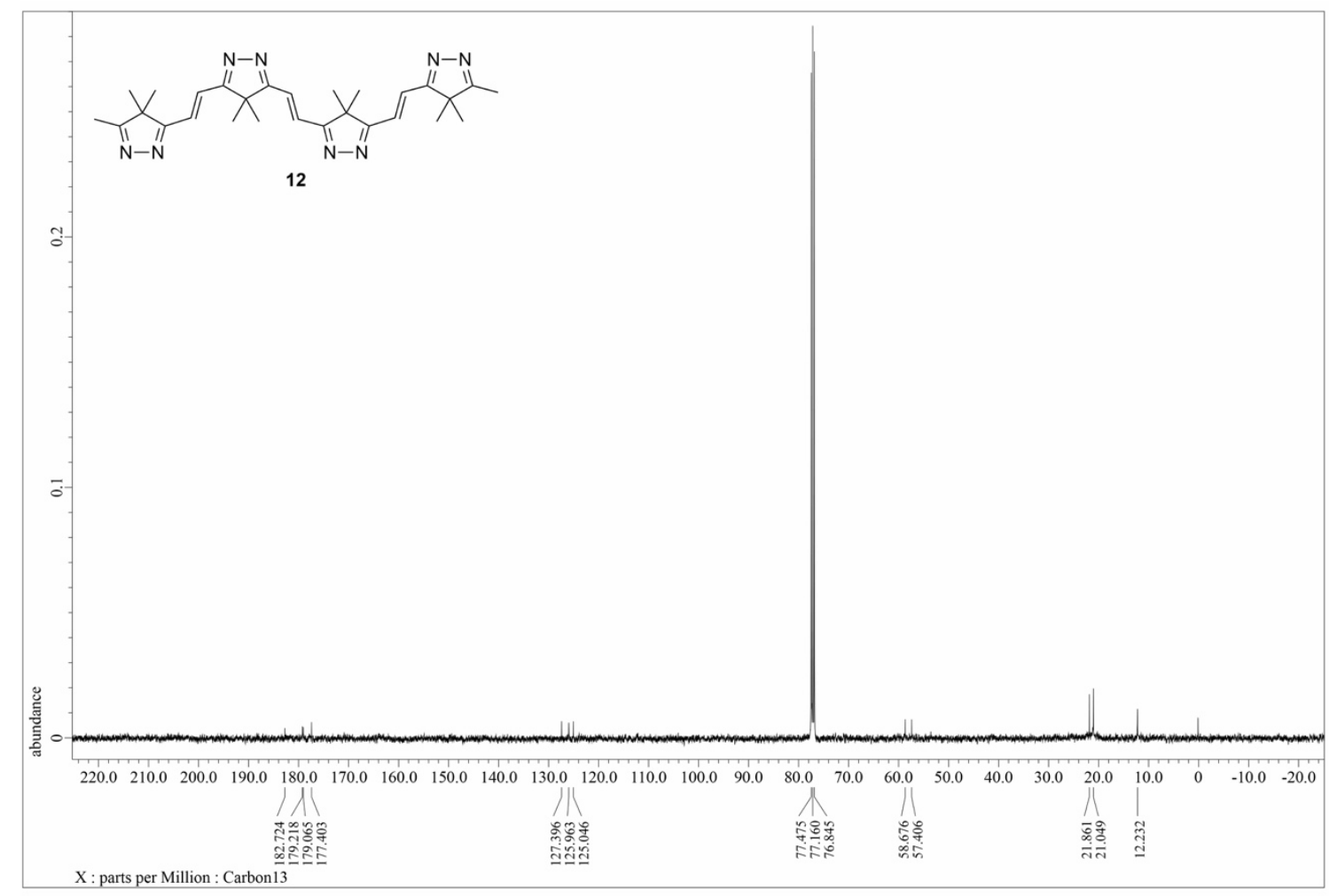

Figure S11. ${ }^{13} \mathrm{C}\left\{{ }^{1} \mathrm{H}\right\}$ NMR spectrum of compound 12 in $\mathrm{CDCl}_{3}$. 


\section{X-ray Structure}

\section{Crystallographic data for compound 5}

$\mathrm{C}_{21} \mathrm{H}_{32} \mathrm{O}_{6}, M=380.46$, crystal size: $0.52 \times 0.22 \times 0.05 \mathrm{~mm}^{3}$, Monoclinic, space group $P 2{ }_{1} / c, a=$ 31.153(6) $\AA, b=6.5006(14) \AA, c=10.454(2) \AA, \beta=96.184(7)^{\circ}, V=2104.7(7) \AA^{3}, Z=4, T=123(2)$ $\mathrm{K}, \mu=0.087 \mathrm{~mm}^{-1}, D_{\text {calc }}=1.201 \mathrm{~g} / \mathrm{cm}^{3}, 1.973^{\circ} \leq \theta \leq 27.484^{\circ}, 2152$ unique reflections out of 4817 with $I>2 \sigma(I), \mathrm{GOF}=1.003, R_{1}=0.0842$ and $w R_{2}=0.2428$ for all data. $\quad$ CCDC number: 1907900 .

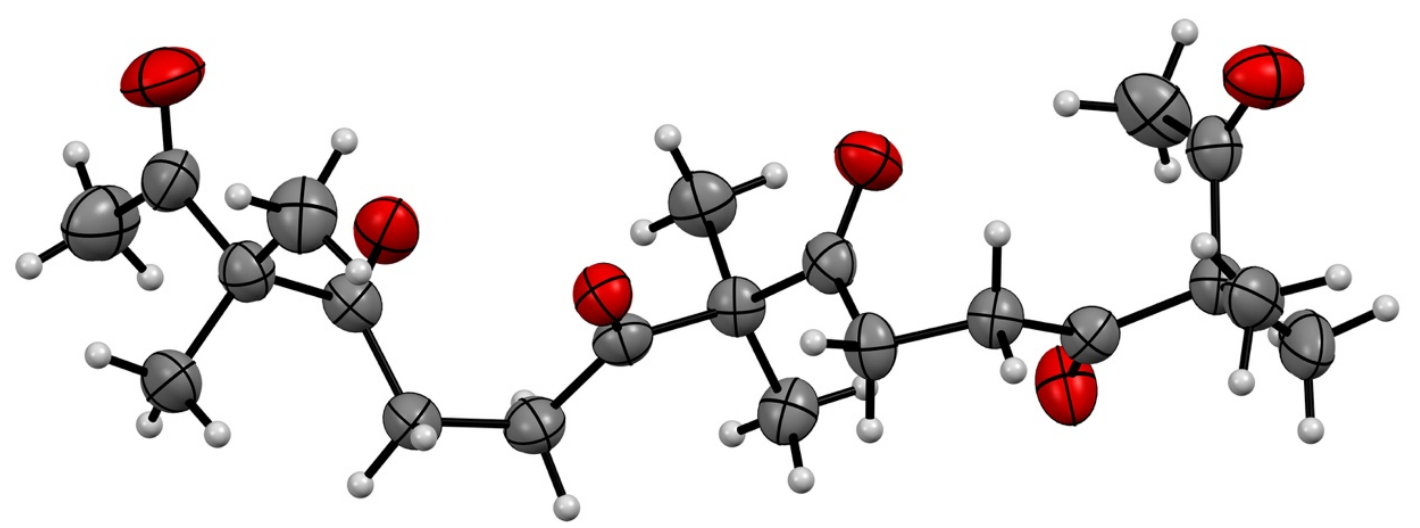

Figure S12. Single crystal X-ray structure of compound 5. 


\section{DFT Calculations}

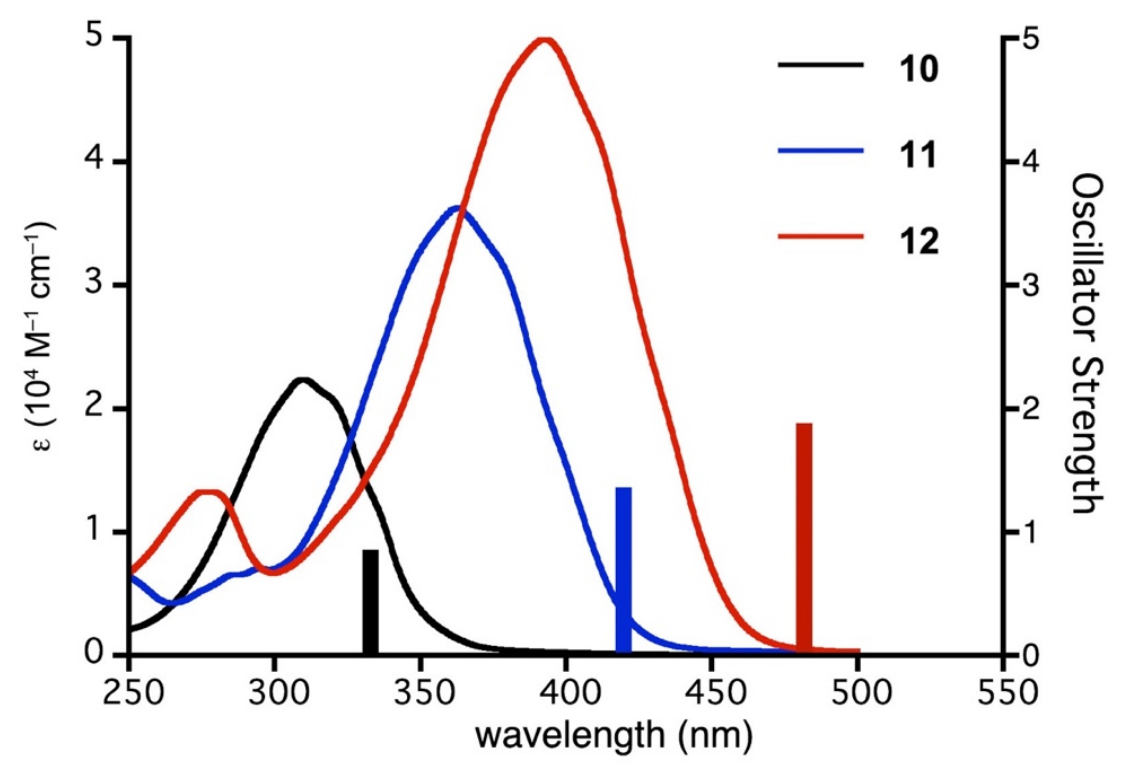

Figure S13. TD-DFT calculated transition (bar) and observed (lines; in $\mathrm{CH}_{2} \mathrm{Cl}_{2}$ ) absorption spectra of conjugated oligoimines 10-12. Characters of the calculated transition bands are listed below.

Transition at $332.92 \mathrm{~nm}$ for 10: HOMO $\rightarrow$ LUMO (99\%), oscillator strength $f=0.7927$

Transition at $419.81 \mathrm{~nm}$ for 11: HOMO $\rightarrow$ LUMO $(100 \%)$, oscillator strength $f=1.2955$

Transition at $481.81 \mathrm{~nm}$ for 12: HOMO $\rightarrow$ LUMO $(100 \%)$, oscillator strength $f=1.8152$

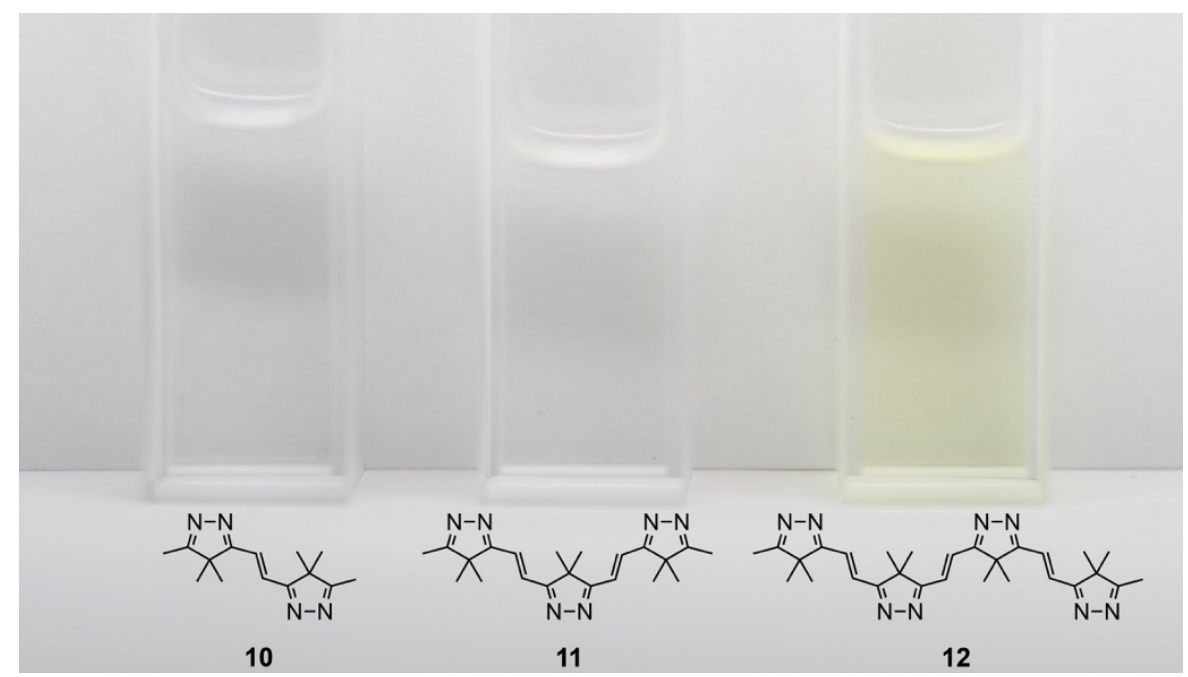

Figure S14. Photograph of dichloromethane solutions of 10-12. 
6mer

$$
\text { , }
$$

$$
\text { 8mer }
$$

\section{2 mer}

$$
\text { : }
$$

\section{6 mer}

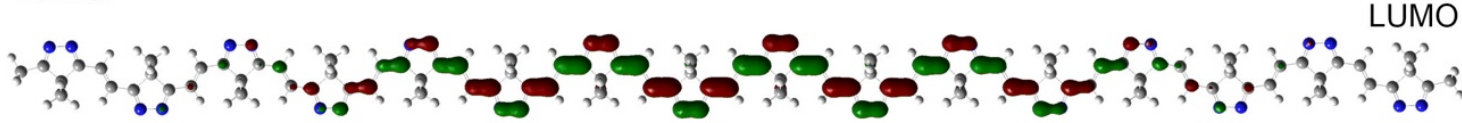

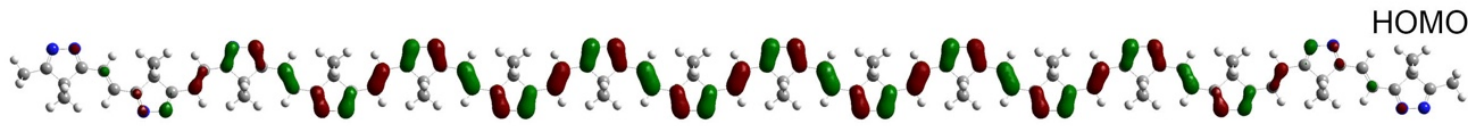

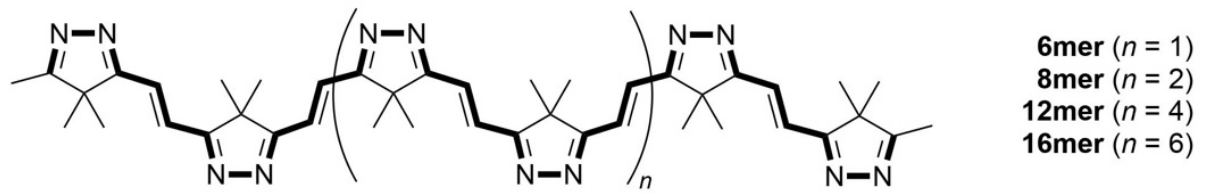

Figure S15. Frontier orbitals for conjugated polyimines 6 16mers calculated at the B3LYP/6-31G* level. 


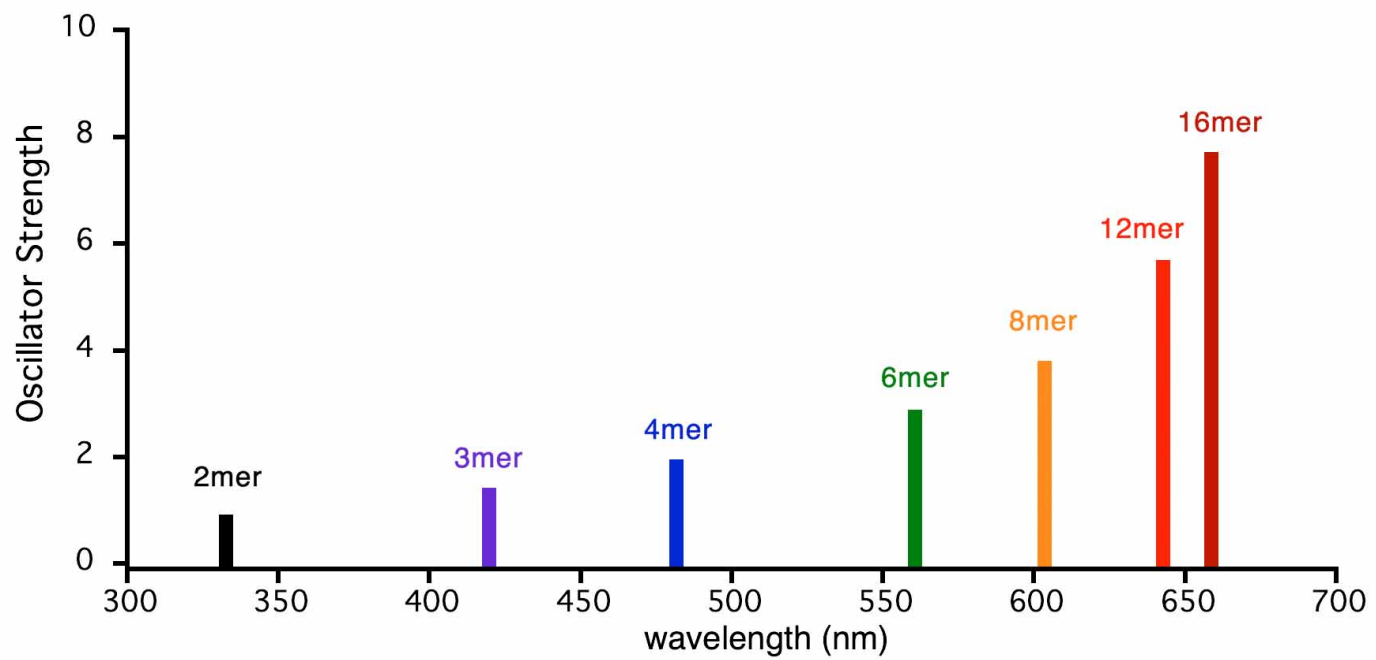

Figure S16. TD-DFT calculated lowest energy transitions for conjugated imines (dimer to hexadecamers) calculated at the B3LYP/6-31G* level. 


\section{Cadmium complex}

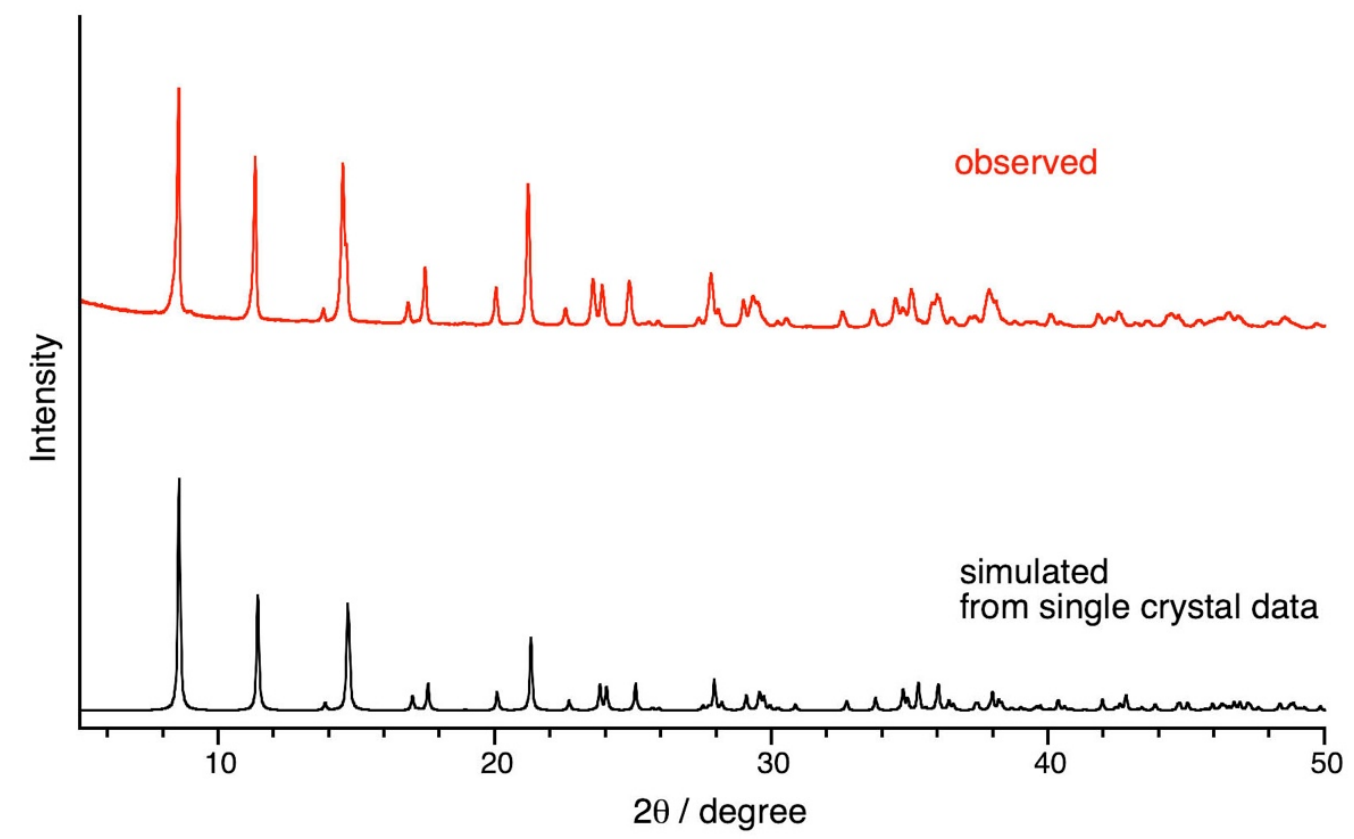

Figure S17. Comparison of the powder X-ray diffraction patterns (red) microcrystals obtained from acetonitrile solution, and (black) simulation pattern based on single crystal analysis. Single crystals were grown from ethanol over 2 weeks at room temperature. 


\section{Synthesis of Polymer Materials}

\section{Polymer 15}

To a 200-mL round bottom flask equipped with a reflux condenser, were added compound $\mathbf{3}$ (10.0 g, $36.7 \mathrm{mmol}$ ), dimethyl sulfone (20.8 g, $220 \mathrm{mmol})$, dimethyl sulfoxide (573 $\mathrm{mg}, 7.34 \mathrm{mmol})$, and silver(I) oxide (10.2 g, $44.0 \mathrm{mmol})$. The reaction mixture was stirred at $100{ }^{\circ} \mathrm{C}$ for $2 \mathrm{~h}$ using an oil bath. After cooling to room temperature, the reaction mixture was passed through a Celite pad using $140 \mathrm{~mL}$ of dichloromethane. The solvent was evaporated, the residue was washed with water (50 $\mathrm{mL} \times 2)$ and then dimethyl sulfone was removed in vacuo $\left(\sim 1 \mathrm{mmHg}, 150^{\circ} \mathrm{C}\right)$. The residue was dissolved in dichloromethane/methanol $(40 \mathrm{~mL}, 1: 1, \mathrm{v} / \mathrm{v})$, and stirred with hydrochloric acid (4.0 $\mathrm{mL}$, $3.0 \mathrm{M}$ ) for $30 \mathrm{~min}$ at room temperature. The solution was neutralized with sat. aq. $\mathrm{NaHCO}_{3}$ and the aqueous layer was extracted with dichloromethane $(50 \mathrm{~mL} \times 2)$. The combined organic layer was washed with brine $(100 \mathrm{~mL})$, and dried over anhydrous $\mathrm{Na}_{2} \mathrm{SO}_{4}$ before evaporating the solvent. The crude residue was chromatographed on a silica gel column (diameter, $4.0 \mathrm{~cm}$; height, $10.0 \mathrm{~cm}$ ) using dichloromethane/methanol $(10: 1, \mathrm{v} / \mathrm{v})$ as eluent to give polymer $15(2.99 \mathrm{~g})$ in $64 \%$ yield. ${ }^{1} \mathrm{H}$ NMR $\left(400 \mathrm{MHz}, \quad \mathrm{CDCl}_{3}, \quad 298 \mathrm{~K}\right): \quad \delta \quad 2.77-2.64$ (ethylene), 2.16-2.14 (acetyl), 1.42-1.34 (dimethylmethylene); ${ }^{13} \mathrm{C}$ NMR (100 MHz, $\left.\mathrm{CDCl}_{3}, 298 \mathrm{~K}\right): \delta \quad$ 207.7-209.6, 62.1-61.7, 32.2, 26.3, 21.8-20.9; IR (ATR, neat): 2979, 2937, 2920, 2875, 1692, 1465, 1388, 1364, $1014 \mathrm{~cm}^{-1}$.

\section{Polymer 16}

Polymer $15(2.00 \mathrm{~g})$ was dissolved in THF $(50 \mathrm{~mL})$ in a 100-mL round-bottom flask equipped with a reflux condenser. Hydrazine monohydrate $(0.96 \mathrm{~mL}, 19.9 \mathrm{mmol})$ was added and the mixture was stirred for $30 \mathrm{~min}$ at $50{ }^{\circ} \mathrm{C}$. After cooling the solution to room temperature, the solvent was evaporated using a rotary evaporator. The residue was dissolved in $\mathrm{CHCl}_{3}(100 \mathrm{~mL})$ and washed with distilled water $(100 \mathrm{~mL})$. The aqueous layer was further extracted with $\mathrm{CHCl}_{3}(100 \mathrm{~mL} \times 2)$, and the combined organic layer was dried over anhydrous $\mathrm{Na}_{2} \mathrm{SO}_{4}$, and the solvent was removed using a rotary evaporator to give crude product. The crude product was washed with $\mathrm{Et}_{2} \mathrm{O}(30 \mathrm{~mL})$ and collected by suction filtration to give polyimine $16(1.83 \mathrm{~g})$ in $94 \%$ yield. ${ }^{1} \mathrm{H}$ NMR (400 MHz, $\mathrm{CDCl}_{3}, 298 \mathrm{~K}$ ): $\delta 2.99-2.94$ (ethylene), 2.16-2.13 (terminal methyl), 1.24-1.16 (dimethylmethylene);

${ }^{13} \mathrm{C}$ NMR (100 MHz, $\left.\mathrm{CDCl}_{3}, 298 \mathrm{~K}\right): \delta \quad 182.2-180.3,60.2-59.8,22.6-22.2,20.6-20.1,12.3-12.1$; IR (ATR, neat): 3281, 2966, 2929, 2911, 2871, 2364, 2356, 2342, 2333, 2325, 1699, 1569, 1463, 1427 , $1385,1362,1099,613 \mathrm{~cm}^{-1}$. 


\section{Polymer 17}

To a solution of polyimine $16(500 \mathrm{mg})$ in 1,4-dioxane $(750 \mathrm{~mL})$ in a 1-L round-bottom flask, was added $p$-chloranil $(2.50 \mathrm{~g}, 10.2 \mathrm{mmol})$. The mixture was refluxed for $5 \mathrm{~h}$ with vigorous stirring. After cooling to room temperature, the resulting precipitate was obtained by suction filtration and rinsed with 1,4-dioxane $(350 \mathrm{~mL})$ and methanol $(250 \mathrm{~mL})$ to give polymer $17(281 \mathrm{mg})$ as a dark brown solid in 57\% yield. IR (ATR, neat): 2973, 2933, 2872, 1687, 1617, 1568, 1547, 1509, 1459, 1368, 1116 , $994,980,614 \mathrm{~cm}^{-1}$. 


\section{Characterization of Polymer Materials}

(a)<smiles>CC(=O)C(C)(C)C(=O)CC(C)(C)C(=O)CC(C)(C)C(=O)C(C)(C)C(=O)C(C)(C)C(C)=O</smiles>

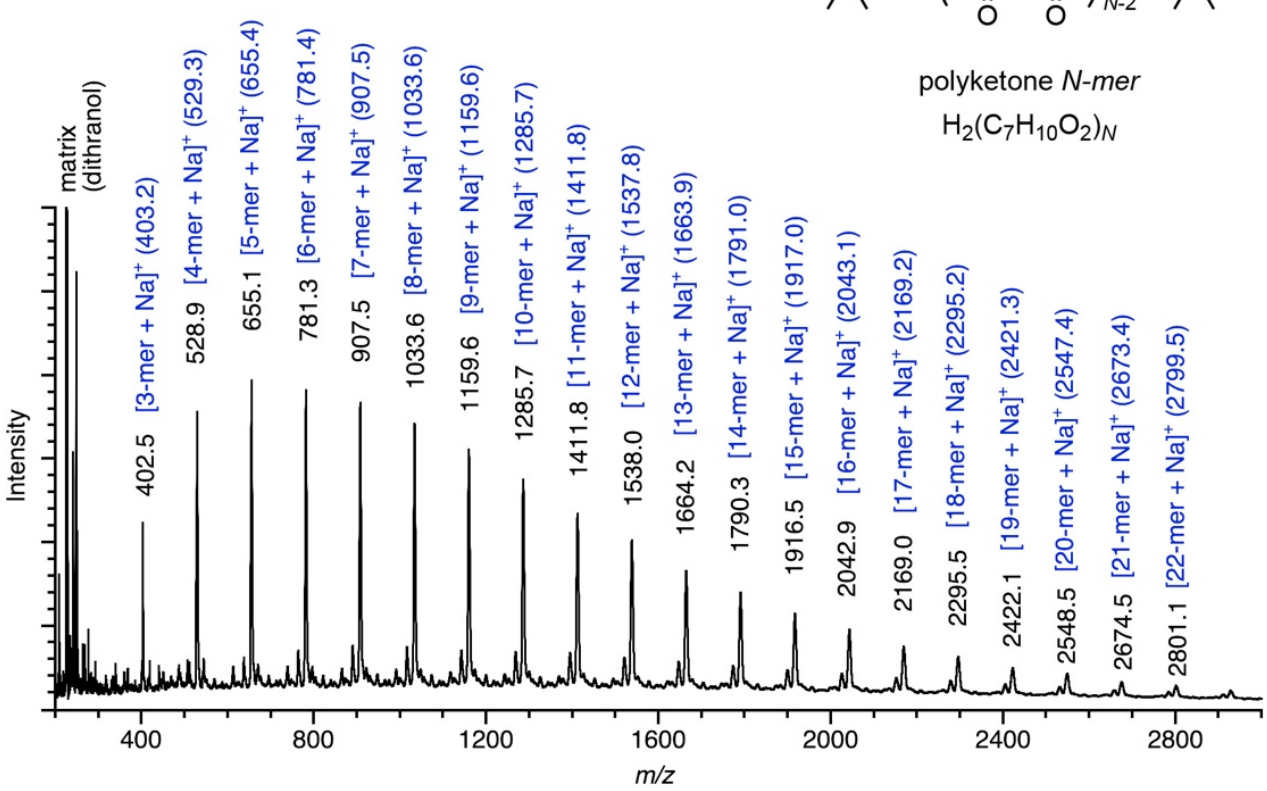

(b)

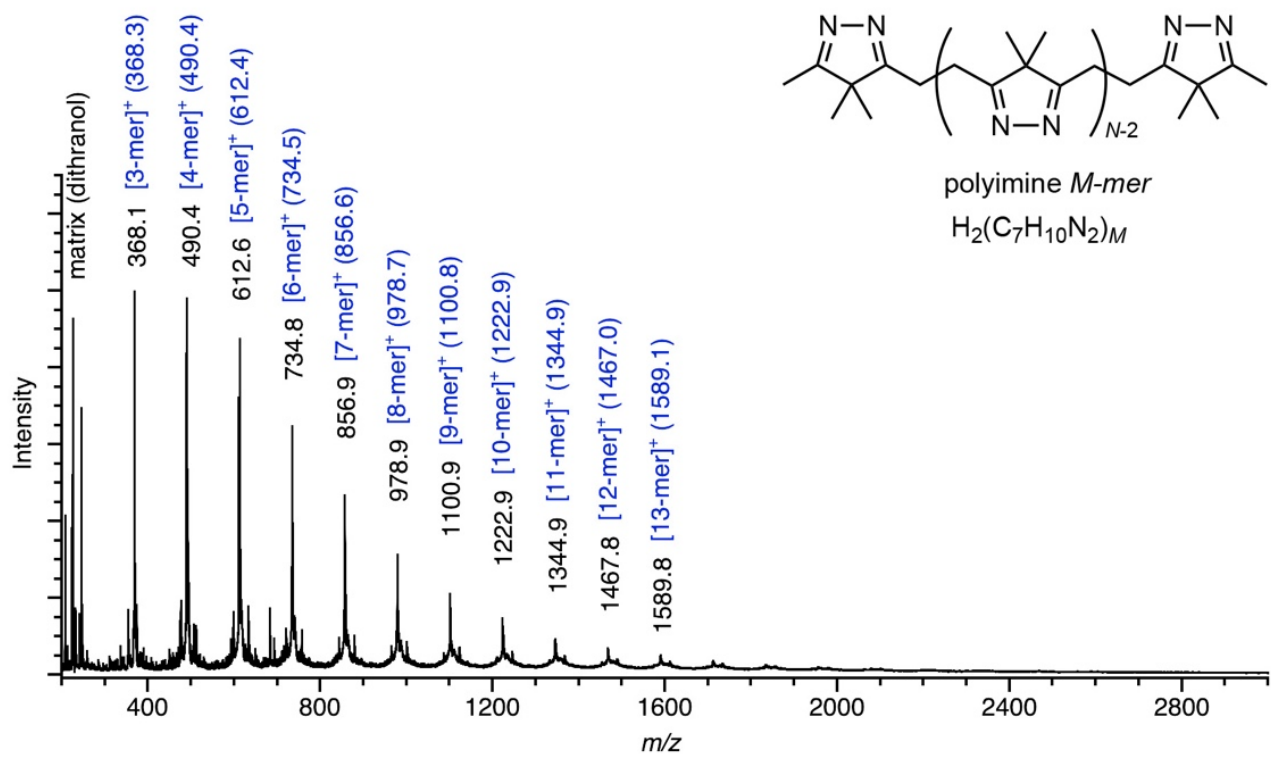

Figure S18. MALDI-TOF-mass spectra of (a) polyketone 15 and (b) polyimine 16 recorded using dithranol as a matrix (black values: observed $m / z$, blue: calculated values for corresponding ions). 


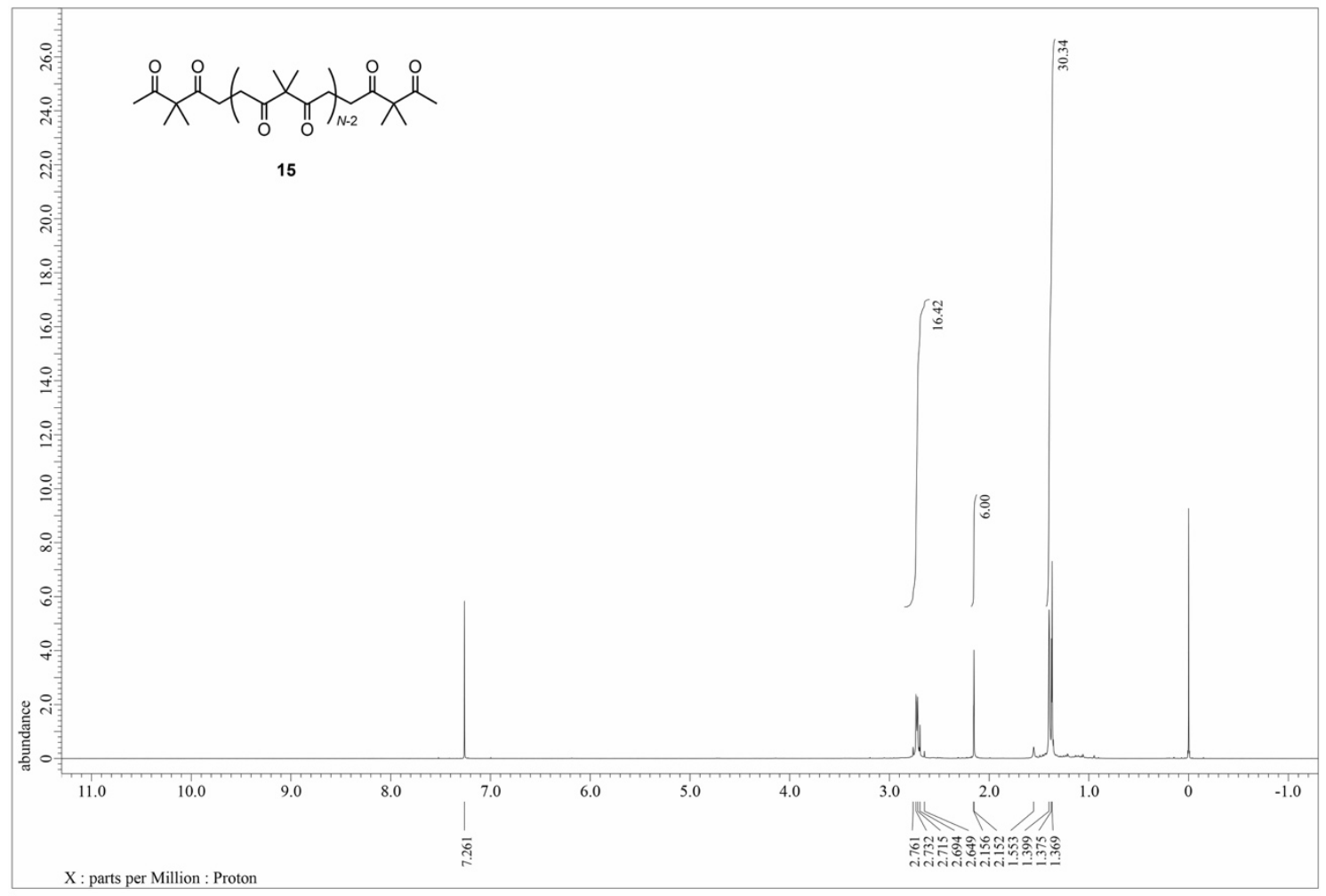

Figure S19. ${ }^{1} \mathrm{H}$ NMR spectra of polymer 15 in $\mathrm{CDCl}_{3}$. The integration ratios of ethylene protons/terminal methyl protons were $16.4: 6$, which indicates the mean degree of polymerization of 5.1. 


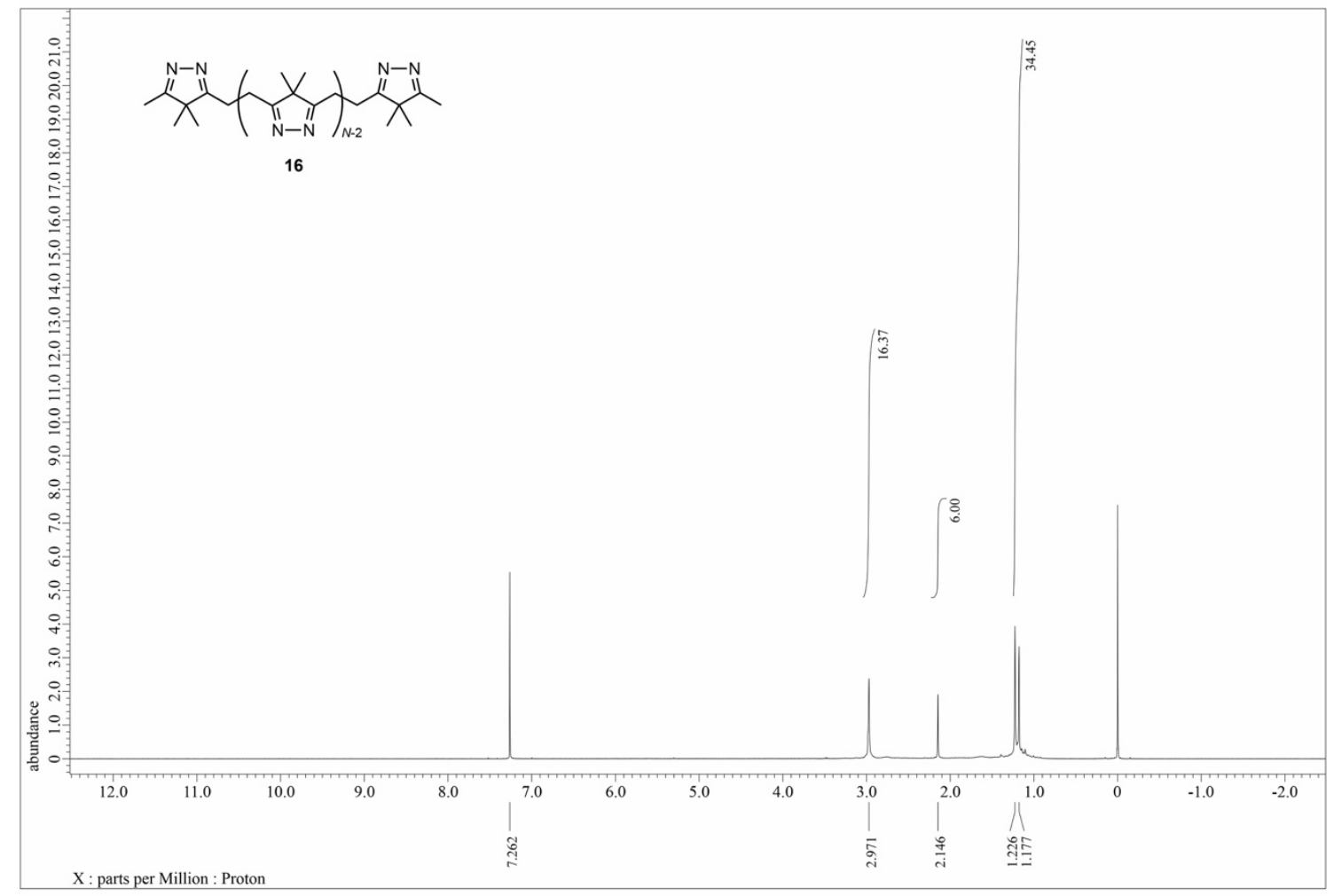

Figure S20. ${ }^{1} \mathrm{H}$ NMR spectra of polymer 16 in $\mathrm{CDCl}_{3}$. The integration ratios of ethylene protons/terminal methyl protons were $16.4: 6$, which indicates the mean degree of polymerization of 5.1. 


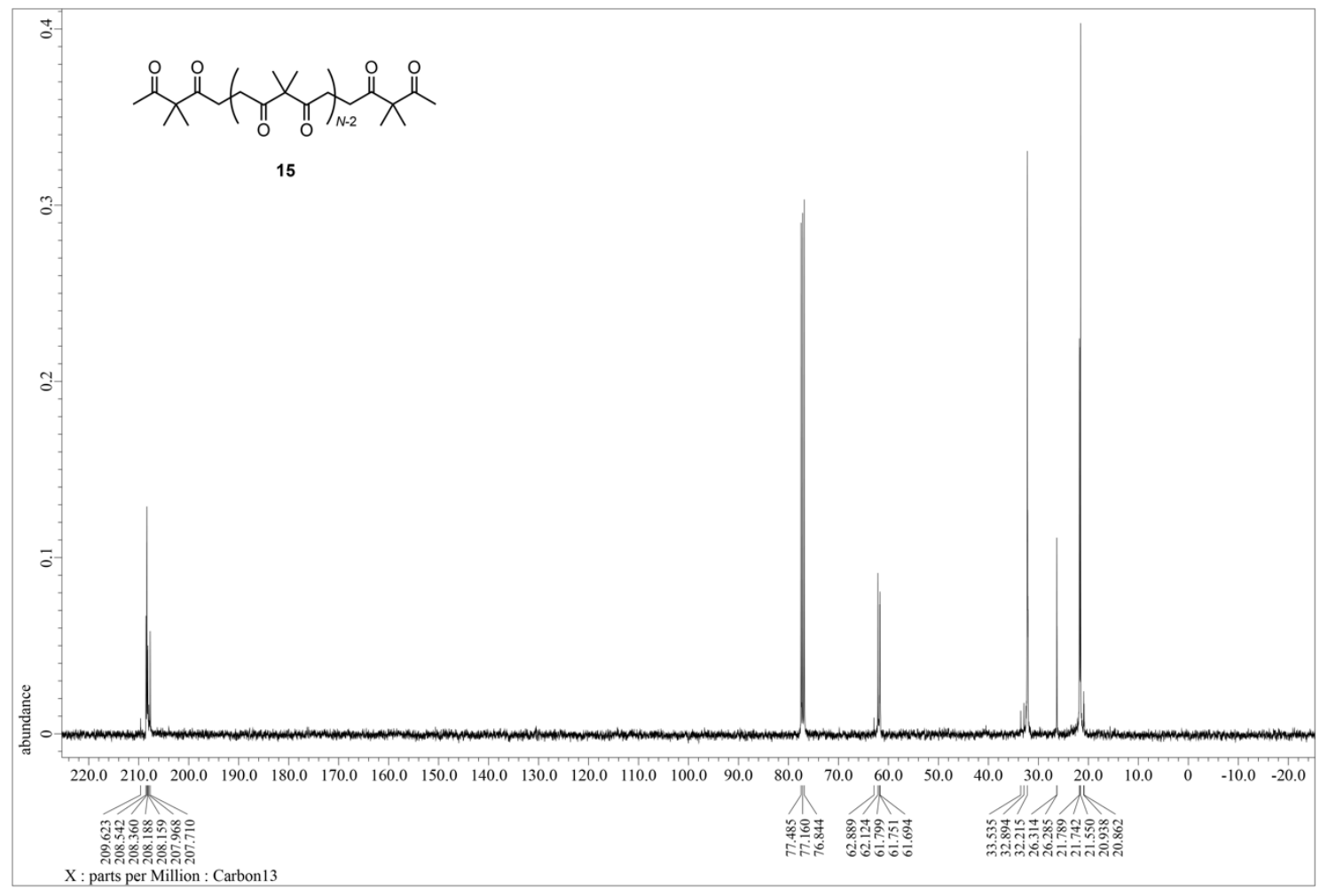

Figure S21. ${ }^{13} \mathrm{C}\left\{{ }^{1} \mathrm{H}\right\}$ NMR spectrum of polymer $\mathbf{1 5}$ in $\mathrm{CDCl}_{3}$.

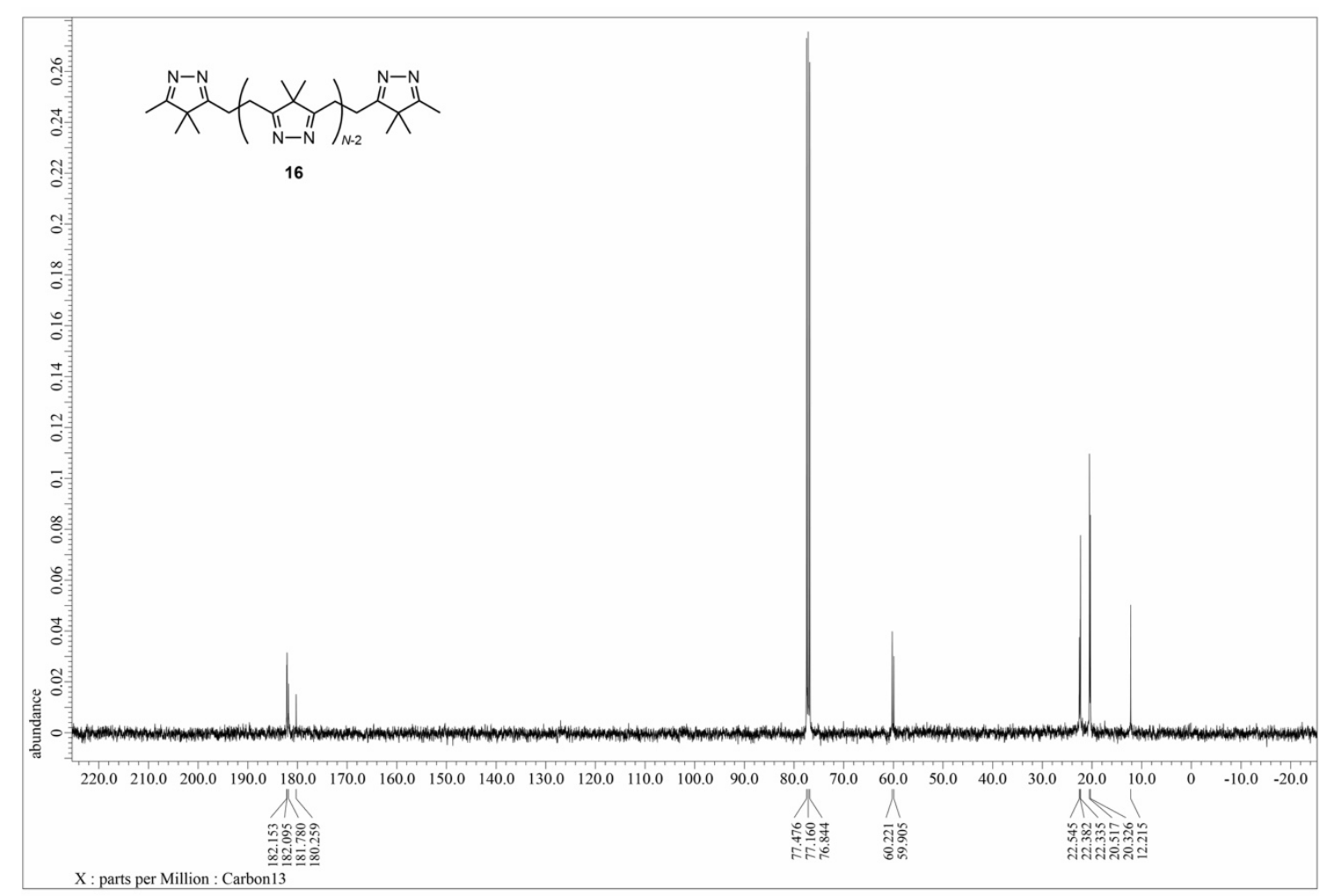

Figure S22. ${ }^{13} \mathrm{C}\left\{{ }^{1} \mathrm{H}\right\}$ NMR spectrum of polymer 16 in $\mathrm{CDCl}_{3}$. 

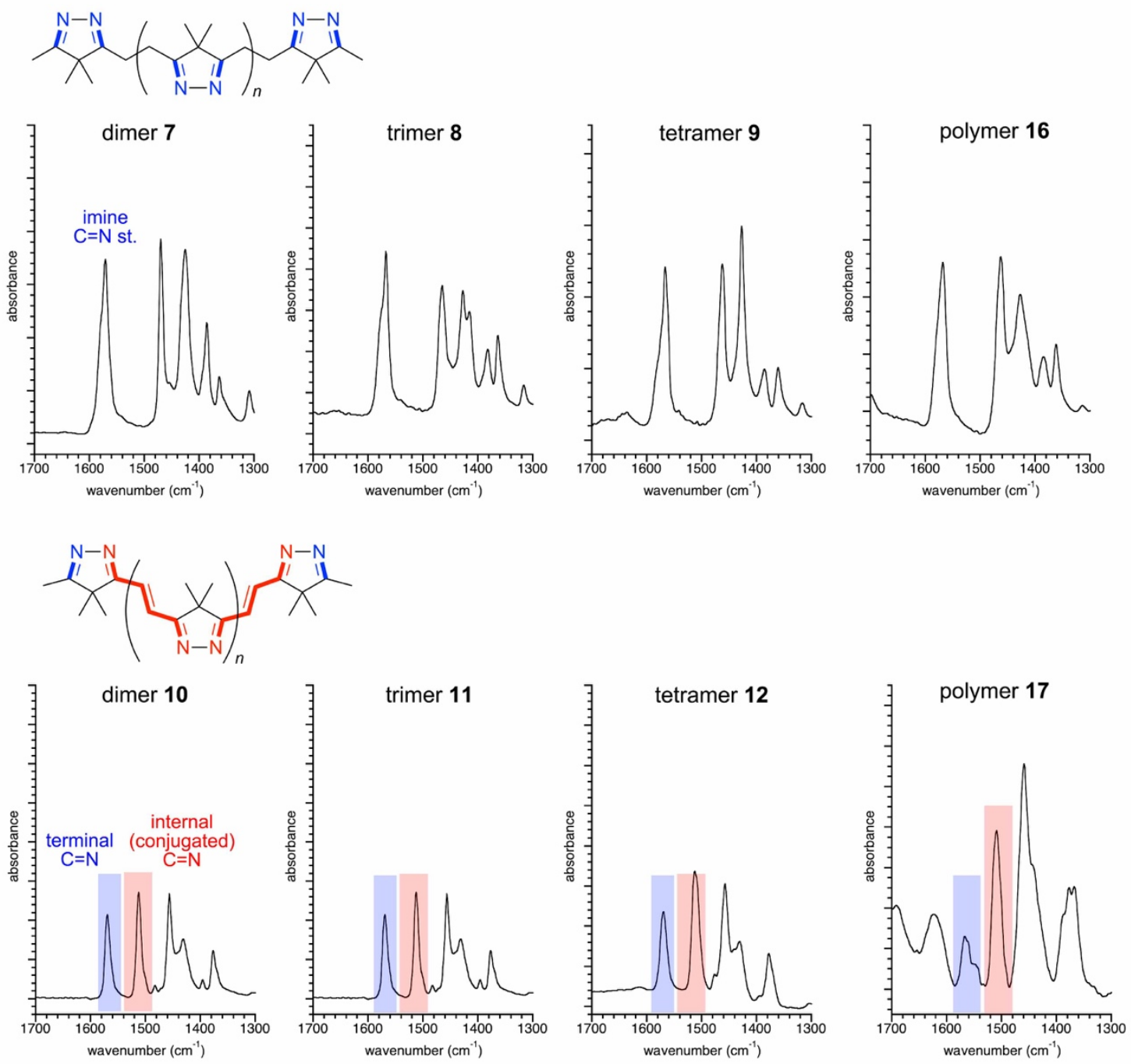

Figure S23. Comparison of IR spectra for ethylene-bridged oligo- and polyimines 7-9 and $\mathbf{1 6}$ with conjugated imines 10-12 and 17. 


\section{Cartesian coordinates of optimized structures}

Conjugated polyimine $2 \mathrm{mer}$

Total Energy $=-764.45643146$ (a.u.)

\begin{tabular}{|c|c|c|c|}
\hline $\mathrm{C}$ & 5.31213420 & -0.42267841 & 0.00000000 \\
\hline $\mathrm{H}$ & 6.04145021 & 0.26715911 & 0.00000000 \\
\hline $\mathrm{H}$ & 5.34450298 & -1.10726820 & -0.82676259 \\
\hline $\mathrm{H}$ & 5.34450298 & -1.10726820 & 0.82676259 \\
\hline $\mathrm{C}$ & 3.97865701 & 0.25787700 & 0.00000000 \\
\hline $\mathrm{C}$ & 2.66027419 & -0.46989938 & 0.00000000 \\
\hline $\mathrm{C}$ & 1.76416457 & 0.74148525 & 0.00000000 \\
\hline $\mathrm{C}$ & 0.26973548 & 0.67005999 & 0.00000000 \\
\hline $\mathrm{N}$ & 3.84627557 & 1.53467659 & 0.00000000 \\
\hline $\mathrm{C}$ & 2.55088837 & -1.16883990 & 1.39753555 \\
\hline $\mathrm{H}$ & 3.29006166 & -1.85691959 & 1.48735958 \\
\hline $\mathrm{H}$ & 1.65116871 & -1.62974247 & 1.47294642 \\
\hline $\mathrm{H}$ & 2.63958207 & -0.48150118 & 2.13802434 \\
\hline $\mathrm{C}$ & 2.55088837 & -1.16883990 & -1.39753555 \\
\hline $\mathrm{H}$ & 2.63958207 & -0.48150118 & -2.13802434 \\
\hline $\mathrm{H}$ & 1.65116871 & -1.62974247 & -1.47294642 \\
\hline $\mathrm{H}$ & 3.29006166 & -1.85691959 & -1.48735958 \\
\hline $\mathrm{N}$ & 2.41800976 & 1.84643276 & 0.00000000 \\
\hline $\mathrm{C}$ & -5.31213420 & 0.42267841 & 0.00000000 \\
\hline $\mathrm{H}$ & -6.04145021 & -0.26715911 & 0.00000000 \\
\hline $\mathrm{H}$ & -5.34450298 & 1.10726820 & 0.82676259 \\
\hline $\mathrm{H}$ & -5.34450298 & 1.10726820 & -0.82676259 \\
\hline $\mathrm{C}$ & -3.97865701 & -0.25787700 & 0.00000000 \\
\hline $\mathrm{C}$ & -2.66027419 & 0.46989938 & 0.00000000 \\
\hline $\mathrm{C}$ & -1.76416457 & -0.74148525 & 0.00000000 \\
\hline $\mathrm{C}$ & -0.26973548 & -0.67005999 & 0.00000000 \\
\hline $\mathrm{N}$ & -3.84627557 & -1.53467659 & 0.00000000 \\
\hline $\mathrm{C}$ & -2.55088837 & 1.16883990 & -1.39753555 \\
\hline
\end{tabular}




$\begin{array}{lrrr}\mathrm{H} & -3.29006166 & 1.85691959 & -1.48735958 \\ \mathrm{H} & -1.65116871 & 1.62974247 & -1.47294642 \\ \mathrm{H} & -2.63958207 & 0.48150118 & -2.13802434 \\ \mathrm{C} & -2.55088837 & 1.16883990 & 1.39753555 \\ \mathrm{H} & -2.63958207 & 0.48150118 & 2.13802434 \\ \mathrm{H} & -1.65116871 & 1.62974247 & 1.47294642 \\ \mathrm{H} & -3.29006166 & 1.85691959 & 1.48735958 \\ \mathrm{~N} & -2.41800976 & -1.84643276 & 0.00000000 \\ \mathrm{H} & 0.36186032 & -1.50874456 & 0.00000000 \\ \mathrm{H} & -0.36186032 & 1.50874456 & 0.00000000\end{array}$

Conjugated polyimine $3 \mathrm{mer}$

Total Energy $=-1145.47276162$ (a.u.)

$\begin{array}{lrrr}\mathrm{C} & 0.00000000 & 3.00901491 & 0.45278807 \\ \mathrm{C} & 0.00000000 & 4.41618146 & 0.80182671 \\ \mathrm{C} & 0.00000000 & 5.55240952 & -0.20428590 \\ \mathrm{C} & 0.00000000 & 6.69169362 & 0.79488873 \\ \mathrm{C} & 0.00000000 & 8.14539070 & 0.45338375 \\ \mathrm{~N} & 0.00000000 & 4.85043626 & 2.02847033 \\ \mathrm{C} & -1.27199949 & 5.54965436 & -1.08132858 \\ \mathrm{H} & -1.29812489 & 4.65873568 & -1.71800685 \\ \mathrm{H} & -1.28782073 & 6.42939010 & -1.73454710 \\ \mathrm{H} & -2.17862200 & 5.55824923 & -0.46788576 \\ \mathrm{C} & 1.27199949 & 5.54965436 & -1.08132858 \\ \mathrm{H} & 2.17862200 & 5.55824923 & -0.46788576 \\ \mathrm{H} & 1.28782073 & 6.42939010 & -1.73454710 \\ \mathrm{H} & 1.29812489 & 4.65873568 & -1.71800685 \\ \mathrm{~N} & 0.00000000 & 6.27473903 & 2.01933071 \\ \mathrm{H} & -0.88186522 & 8.41441712 & -0.14287476 \\ \mathrm{C} & 0.00000000 & -8.14539070 & 0.45338375 \\ \mathrm{H} & 0.00000000 & -8.73994675 & 1.36986332 \\ \mathrm{H} & 0.88186522 & -8.41441712 & -0.14287476\end{array}$




\begin{tabular}{|c|c|c|c|}
\hline $\mathrm{H}$ & -0.88186522 & -8.41441712 & -0.14287476 \\
\hline $\mathrm{C}$ & 0.00000000 & -6.69169362 & 0.79488873 \\
\hline $\mathrm{C}$ & 0.00000000 & -5.55240952 & -0.20428590 \\
\hline $\mathrm{C}$ & 0.00000000 & -4.41618146 & 0.80182671 \\
\hline $\mathrm{C}$ & 0.00000000 & -3.00901491 & 0.45278807 \\
\hline $\mathrm{N}$ & 0.00000000 & -6.27473903 & 2.01933071 \\
\hline $\mathrm{C}$ & -1.27199949 & -5.54965436 & -1.08132858 \\
\hline $\mathrm{H}$ & -1.28782073 & -6.42939010 & -1.73454710 \\
\hline $\mathrm{H}$ & -1.29812489 & -4.65873568 & -1.71800685 \\
\hline $\mathrm{H}$ & -2.17862200 & -5.55824923 & -0.46788576 \\
\hline $\mathrm{C}$ & 1.27199949 & -5.54965436 & -1.08132858 \\
\hline $\mathrm{H}$ & 2.17862200 & -5.55824923 & -0.46788576 \\
\hline $\mathrm{H}$ & 1.29812489 & -4.65873568 & -1.71800685 \\
\hline $\mathrm{H}$ & 1.28782073 & -6.42939010 & -1.73454710 \\
\hline $\mathrm{N}$ & 0.00000000 & -4.85043626 & 2.02847033 \\
\hline $\mathrm{C}$ & 0.00000000 & 2.54260395 & -0.81917520 \\
\hline $\mathrm{C}$ & 0.00000000 & 1.13763089 & -1.16308587 \\
\hline $\mathrm{C}$ & 0.00000000 & 0.00000000 & -0.16171256 \\
\hline $\mathrm{C}$ & 0.00000000 & -1.13763089 & -1.16308587 \\
\hline $\mathrm{C}$ & 0.00000000 & -2.54260395 & -0.81917520 \\
\hline $\mathrm{N}$ & 0.00000000 & 0.70151215 & -2.39575665 \\
\hline $\mathrm{C}$ & 1.27248319 & -0.00000000 & 0.71644390 \\
\hline $\mathrm{H}$ & 1.29205409 & 0.88580939 & 1.36045547 \\
\hline $\mathrm{H}$ & 1.29205409 & -0.88580939 & 1.36045547 \\
\hline $\mathrm{H}$ & 2.17915799 & -0.00000000 & 0.10347976 \\
\hline $\mathrm{C}$ & -1.27248319 & -0.00000000 & 0.71644390 \\
\hline $\mathrm{H}$ & -2.17915799 & -0.00000000 & 0.10347976 \\
\hline $\mathrm{H}$ & -1.29205409 & -0.88580939 & 1.36045547 \\
\hline $\mathrm{H}$ & -1.29205409 & 0.88580939 & 1.36045547 \\
\hline $\mathrm{N}$ & 0.00000000 & -0.70151215 & -2.39575665 \\
\hline $\mathrm{H}$ & 0.00000000 & -3.26284407 & -1.63398052 \\
\hline $\mathrm{H}$ & 0.00000000 & -2.28731436 & 1.26642020 \\
\hline $\mathrm{H}$ & -0.00000000 & 2.28731436 & 1.26642020 \\
\hline
\end{tabular}




$\begin{array}{rrrr}\mathrm{H} & -0.00000000 & 3.26284407 & -1.63398052 \\ \mathrm{H} & -0.00000000 & 8.73994675 & 1.36986332 \\ \mathrm{H} & 0.88186522 & 8.41441712 & -0.14287476\end{array}$

Conjugated polyimine $4 \mathrm{mer}$

Total Energy $=-1526.48921317$ (a.u.)

$\begin{array}{lrrr}\mathrm{C} & 0.44611292 & 0.51164638 & 0.00000000 \\ \mathrm{C} & 0.14730845 & 1.92370888 & 0.00000000 \\ \mathrm{C} & -1.19569866 & 2.63988232 & 0.00000000 \\ \mathrm{C} & -0.64740089 & 4.05945856 & 0.00000000 \\ \mathrm{C} & -1.34711213 & 5.32287240 & 0.00000000 \\ \mathrm{~N} & 1.14980441 & 2.76759056 & 0.00000000 \\ \mathrm{C} & -2.00474490 & 2.34046861 & 1.28553835 \\ \mathrm{H} & -2.30114661 & 1.28839124 & 1.32247979 \\ \mathrm{H} & -2.91237751 & 2.94944991 & 1.32427636 \\ \mathrm{H} & -1.41160958 & 2.55779173 & 2.17940572 \\ \mathrm{C} & -2.00474490 & 2.34046861 & -1.28553835 \\ \mathrm{H} & -1.41160958 & 2.55779173 & -2.17940572 \\ \mathrm{H} & -2.91237751 & 2.94944991 & -1.32427636 \\ \mathrm{H} & -2.30114661 & 1.28839124 & -1.32247979 \\ \mathrm{~N} & -2.66258520 & 4.07689847 & 0.00000000 \\ \mathrm{C} & -4.31432557 & 10.41347815 & 0.00000000 \\ \mathrm{H} & -5.37822481 & 10.66100483 & 0.00000000 \\ \mathrm{H} & -2.84537243 & 10.86878501 & 0.88180686 \\ \mathrm{H} & -3.84537243 & 10.86878501 & -0.88180686 \\ \mathrm{C} & -4.14396094 & 8.92979215 & 0.00000000 \\ \mathrm{C} & -2.6896606737 & 8.19711746 & 0.00000000 \\ \mathrm{C} & -5.15654706 & 8.12554370 & 0.00000000 \\ \mathrm{C} & 8.00474490 & 8.51823021 & -1.27793623 \\ \mathrm{H} & 9.57734460 & -1.292996661\end{array}$




\begin{tabular}{|c|c|c|c|}
\hline $\mathrm{H}$ & -1.08521760 & 7.92715959 & -1.32026866 \\
\hline $\mathrm{H}$ & -2.58815889 & 8.30357036 & -2.17904603 \\
\hline $\mathrm{C}$ & -2.00474490 & 8.51823021 & 1.27793623 \\
\hline $\mathrm{H}$ & -2.58815889 & 8.30357036 & 2.17904603 \\
\hline $\mathrm{H}$ & -1.08521760 & 7.92715959 & 1.32026866 \\
\hline $\mathrm{H}$ & -1.72475988 & 9.57734460 & 1.29299661 \\
\hline $\mathrm{N}$ & -4.68348409 & 6.78541951 & 0.00000000 \\
\hline $\mathrm{H}$ & -3.36969679 & 4.66697421 & 0.00000000 \\
\hline $\mathrm{H}$ & -0.67151935 & 6.17329812 & 0.00000000 \\
\hline $\mathrm{C}$ & 4.31432557 & -10.41347815 & 0.00000000 \\
\hline $\mathrm{H}$ & 5.37822481 & -10.66100483 & 0.00000000 \\
\hline $\mathrm{H}$ & 3.84537243 & -10.86878501 & -0.88180686 \\
\hline $\mathrm{H}$ & 3.84537243 & -10.86878501 & 0.88180686 \\
\hline $\mathrm{C}$ & 4.14396094 & -8.92979215 & 0.00000000 \\
\hline $\mathrm{C}$ & 2.81406737 & -8.19711746 & 0.00000000 \\
\hline $\mathrm{C}$ & 3.37936652 & -6.78568241 & 0.00000000 \\
\hline $\mathrm{C}$ & 2.68966061 & -5.51359105 & 0.00000000 \\
\hline $\mathrm{N}$ & 5.15654706 & -8.12554370 & 0.00000000 \\
\hline $\mathrm{C}$ & 2.00474490 & -8.51823021 & 1.27793623 \\
\hline $\mathrm{H}$ & 1.72475988 & -9.57734460 & 1.29299661 \\
\hline $\mathrm{H}$ & 1.08521760 & -7.92715959 & 1.32026866 \\
\hline $\mathrm{H}$ & 2.58815889 & -8.30357036 & 2.17904603 \\
\hline $\mathrm{C}$ & 2.00474490 & -8.51823021 & -1.27793623 \\
\hline $\mathrm{H}$ & 2.58815889 & -8.30357036 & -2.17904603 \\
\hline $\mathrm{H}$ & 1.08521760 & -7.92715959 & -1.32026866 \\
\hline $\mathrm{H}$ & 1.72475988 & -9.57734460 & -1.29299661 \\
\hline $\mathrm{N}$ & 4.68348409 & -6.78541951 & 0.00000000 \\
\hline $\mathrm{C}$ & -0.44611292 & -0.51164638 & 0.00000000 \\
\hline $\mathrm{C}$ & -0.14730845 & -1.92370888 & 0.00000000 \\
\hline $\mathrm{C}$ & 1.19569866 & -2.63988232 & 0.00000000 \\
\hline $\mathrm{C}$ & 0.64740089 & -4.05945856 & 0.00000000 \\
\hline $\mathrm{C}$ & 1.34711213 & -5.32287240 & 0.00000000 \\
\hline $\mathrm{N}$ & -1.14980441 & -2.76759056 & 0.00000000 \\
\hline
\end{tabular}




$\begin{array}{lrrr}\mathrm{C} & 2.00474490 & -2.34046861 & -1.28553835 \\ \mathrm{H} & 2.30114661 & -1.28839124 & -1.32247979 \\ \mathrm{H} & 2.91237751 & -2.94944991 & -1.32427636 \\ \mathrm{H} & 1.41160958 & -2.55779173 & -2.17940572 \\ \mathrm{C} & 2.00474490 & -2.34046861 & 1.28553835 \\ \mathrm{H} & 1.41160958 & -2.55779173 & 2.17940572 \\ \mathrm{H} & 2.91237751 & -2.94944991 & 1.32427636 \\ \mathrm{H} & 2.30114661 & -1.28839124 & 1.32247979 \\ \mathrm{~N} & -0.66258520 & -4.07689847 & 0.00000000 \\ \mathrm{H} & 0.67151935 & -6.17329812 & 0.00000000 \\ \mathrm{H} & 3.36969679 & -4.66697421 & 0.00000000 \\ \mathrm{H} & 1.51350915 & 0.31220406 & 0.00000000 \\ \mathrm{H} & -1.51350915 & -0.31220406 & 0.00000000\end{array}$

Conjugated polyimine $6 \mathrm{mer}$

Total Energy $=-2288.52192226$ (a.u.)

$\begin{array}{lrrr}\mathrm{C} & 5.48598805 & 16.14256145 & 0.00000000 \\ \mathrm{C} & 4.65422516 & 14.90228725 & 0.00000000 \\ \mathrm{C} & 5.19098226 & 13.48211733 & 0.00000000 \\ \mathrm{C} & 3.84483709 & 12.77476520 & 0.00000000 \\ \mathrm{C} & 3.54247591 & 11.35991129 & 0.00000000 \\ \mathrm{~N} & 3.36177981 & 14.94727769 & 0.00000000 \\ \mathrm{C} & 6.01524987 & 13.20216733 & 1.27796458 \\ \mathrm{H} & 6.91347157 & 13.82922316 & 1.29491755 \\ \mathrm{H} & 6.33525324 & 12.15693192 & 1.31896283 \\ \mathrm{H} & 5.43089642 & 13.41393729 & 2.17910376 \\ \mathrm{C} & 6.01524987 & 13.20216733 & -1.27796458 \\ \mathrm{H} & 5.43089642 & 13.41393729 & -2.17910376 \\ \mathrm{H} & 6.33525324 & 12.15693192 & -1.31896283 \\ \mathrm{H} & 6.91347157 & 13.82922316 & -1.29491755 \\ \mathrm{~N} & 2.85250449 & 13.62120199 & 0.00000000 \\ \mathrm{C} & 2.66710595 & 5.52275746 & 0.00000000\end{array}$




\begin{tabular}{|c|c|c|c|}
\hline $\mathrm{C}$ & 3.35920166 & 6.78875815 & 0.00000000 \\
\hline $\mathrm{C}$ & 2.80585029 & 8.20622878 & 0.00000000 \\
\hline $\mathrm{C}$ & 4.14633793 & 8.92720881 & 0.00000000 \\
\hline $\mathrm{C}$ & 4.43788650 & 10.34147766 & 0.00000000 \\
\hline $\mathrm{N}$ & 4.66955515 & 6.77661760 & 0.00000000 \\
\hline $\mathrm{C}$ & 1.99604333 & 8.50364640 & -1.28552342 \\
\hline $\mathrm{H}$ & 1.08931224 & 7.89328971 & -1.32387039 \\
\hline $\mathrm{H}$ & 1.69800126 & 9.55515774 & -1.32262918 \\
\hline $\mathrm{H}$ & 2.58928077 & 8.28708172 & -2.17947543 \\
\hline $\mathrm{C}$ & 1.99604333 & 8.50364640 & 1.28552342 \\
\hline $\mathrm{H}$ & 2.58928077 & 8.28708172 & 2.17947543 \\
\hline $\mathrm{H}$ & 1.69800126 & 9.55515774 & 1.32262918 \\
\hline $\mathrm{H}$ & 1.08931224 & 7.89328971 & 1.32387039 \\
\hline $\mathrm{N}$ & 5.15207768 & 8.08711178 & 0.00000000 \\
\hline $\mathrm{H}$ & 5.50425144 & 10.54720278 & 0.00000000 \\
\hline $\mathrm{H}$ & 2.47531149 & 11.15930697 & 0.00000000 \\
\hline $\mathrm{H}$ & 4.83572296 & 17.02013985 & 0.00000000 \\
\hline $\mathrm{C}$ & -2.66710595 & -5.52275746 & 0.00000000 \\
\hline $\mathrm{C}$ & -3.35920166 & -6.78875815 & 0.00000000 \\
\hline $\mathrm{C}$ & -2.80585029 & -8.20622878 & 0.00000000 \\
\hline $\mathrm{C}$ & -4.14633793 & -8.92720881 & 0.00000000 \\
\hline $\mathrm{C}$ & -4.43788650 & -10.34147766 & 0.00000000 \\
\hline $\mathrm{N}$ & -4.66955515 & -6.77661760 & 0.00000000 \\
\hline $\mathrm{C}$ & -1.99604333 & -8.50364640 & 1.28552342 \\
\hline $\mathrm{H}$ & -1.08931224 & -7.89328971 & 1.32387039 \\
\hline $\mathrm{H}$ & -1.69800126 & -9.55515774 & 1.32262918 \\
\hline $\mathrm{H}$ & -2.58928077 & -8.28708172 & 2.17947543 \\
\hline $\mathrm{C}$ & -1.99604333 & -8.50364640 & -1.28552342 \\
\hline $\mathrm{H}$ & -2.58928077 & -8.28708172 & -2.17947543 \\
\hline $\mathrm{H}$ & -1.69800126 & -9.55515774 & -1.32262918 \\
\hline $\mathrm{H}$ & -1.08931224 & -7.89328971 & -1.32387039 \\
\hline $\mathrm{N}$ & -5.15207768 & -8.08711178 & 0.00000000 \\
\hline $\mathrm{C}$ & -5.48598805 & -16.14256145 & 0.00000000 \\
\hline
\end{tabular}




\begin{tabular}{|c|c|c|c|}
\hline $\mathrm{H}$ & -4.83572296 & -17.02013985 & 0.00000000 \\
\hline $\mathrm{H}$ & -6.13804555 & -16.18576724 & 0.88185790 \\
\hline $\mathrm{H}$ & -6.13804555 & -16.18576724 & -0.88185790 \\
\hline $\mathrm{C}$ & -4.65422516 & -14.90228725 & 0.00000000 \\
\hline $\mathrm{C}$ & -5.19098226 & -13.48211733 & 0.00000000 \\
\hline $\mathrm{C}$ & -3.84483709 & -12.77476520 & 0.00000000 \\
\hline $\mathrm{C}$ & -3.54247591 & -11.35991129 & 0.00000000 \\
\hline $\mathrm{N}$ & -3.36177981 & -14.94727769 & 0.00000000 \\
\hline $\mathrm{C}$ & -6.01524987 & -13.20216733 & -1.27796458 \\
\hline $\mathrm{H}$ & -6.91347157 & -13.82922316 & -1.29491755 \\
\hline $\mathrm{H}$ & -6.33525324 & -12.15693192 & -1.31896283 \\
\hline $\mathrm{H}$ & -5.43089642 & -13.41393729 & -2.17910376 \\
\hline $\mathrm{C}$ & -6.01524987 & -13.20216733 & 1.27796458 \\
\hline $\mathrm{H}$ & -5.43089642 & -13.41393729 & 2.17910376 \\
\hline $\mathrm{H}$ & -6.33525324 & -12.15693192 & 1.31896283 \\
\hline $\mathrm{H}$ & -6.91347157 & -13.82922316 & 1.29491755 \\
\hline $\mathrm{N}$ & -2.85250449 & -13.62120199 & 0.00000000 \\
\hline $\mathrm{H}$ & -2.47531149 & -11.15930697 & 0.00000000 \\
\hline $\mathrm{H}$ & -5.50425144 & -10.54720278 & 0.00000000 \\
\hline $\mathrm{C}$ & 1.32327959 & 5.32719006 & 0.00000000 \\
\hline $\mathrm{C}$ & 0.63176221 & 4.06124283 & 0.00000000 \\
\hline $\mathrm{C}$ & 1.18595482 & 2.64390738 & 0.00000000 \\
\hline $\mathrm{C}$ & -0.15417547 & 1.92244732 & 0.00000000 \\
\hline $\mathrm{C}$ & -0.44809203 & 0.51021819 & 0.00000000 \\
\hline $\mathrm{N}$ & -0.67929447 & 4.07251291 & 0.00000000 \\
\hline $\mathrm{C}$ & 1.99604333 & 2.34723680 & 1.28557149 \\
\hline $\mathrm{H}$ & 2.90184597 & 2.95889882 & 1.32410963 \\
\hline $\mathrm{H}$ & 2.29580916 & 1.29615802 & 1.32241396 \\
\hline $\mathrm{H}$ & 1.40250081 & 2.56271178 & 2.17956390 \\
\hline $\mathrm{C}$ & 1.99604333 & 2.34723680 & -1.28557149 \\
\hline $\mathrm{H}$ & 1.40250081 & 2.56271178 & -2.17956390 \\
\hline $\mathrm{H}$ & 2.29580916 & 1.29615802 & -1.32241396 \\
\hline $\mathrm{H}$ & 2.90184597 & 2.95889882 & -1.32410963 \\
\hline
\end{tabular}




\begin{tabular}{|c|c|c|c|}
\hline $\mathrm{N}$ & -1.16038298 & 2.76305199 & 0.00000000 \\
\hline $\mathrm{C}$ & -1.32327959 & -5.32719006 & 0.00000000 \\
\hline $\mathrm{C}$ & -0.63176221 & -4.06124283 & 0.00000000 \\
\hline $\mathrm{C}$ & -1.18595482 & -2.64390738 & 0.00000000 \\
\hline $\mathrm{C}$ & 0.15417547 & -1.92244732 & 0.00000000 \\
\hline $\mathrm{C}$ & 0.44809203 & -0.51021819 & 0.00000000 \\
\hline $\mathrm{N}$ & 0.67929447 & -4.07251291 & 0.00000000 \\
\hline $\mathrm{C}$ & -1.99604333 & -2.34723680 & -1.28557149 \\
\hline $\mathrm{H}$ & -2.90184597 & -2.95889882 & -1.32410963 \\
\hline $\mathrm{H}$ & -2.29580916 & -1.29615802 & -1.32241396 \\
\hline $\mathrm{H}$ & -1.40250081 & -2.56271178 & -2.17956390 \\
\hline $\mathrm{C}$ & -1.99604333 & -2.34723680 & 1.28557149 \\
\hline $\mathrm{H}$ & -1.40250081 & -2.56271178 & 2.17956390 \\
\hline $\mathrm{H}$ & -2.29580916 & -1.29615802 & 1.32241396 \\
\hline $\mathrm{H}$ & -2.90184597 & -2.95889882 & 1.32410963 \\
\hline $\mathrm{N}$ & 1.16038298 & -2.76305199 & 0.00000000 \\
\hline $\mathrm{H}$ & 1.51470248 & -0.30688793 & 0.00000000 \\
\hline $\mathrm{H}$ & -1.51470248 & 0.30688793 & 0.00000000 \\
\hline $\mathrm{H}$ & -3.34711499 & -4.67617630 & 0.00000000 \\
\hline $\mathrm{H}$ & -0.64333086 & -6.17377317 & 0.00000000 \\
\hline $\mathrm{H}$ & 3.34711499 & 4.67617630 & 0.00000000 \\
\hline $\mathrm{H}$ & 0.64333086 & 6.17377317 & 0.00000000 \\
\hline $\mathrm{H}$ & 6.13804555 & 16.18576724 & 0.88185790 \\
\hline $\mathrm{H}$ & 6.13804555 & 16.18576724 & -0.88185790 \\
\hline
\end{tabular}

Conjugated polyimine $8 \mathrm{mer}$

Total Energy $=-3050.55450668$ (a.u.)

$\begin{array}{lrrr}\mathrm{C} & 0.78011576 & 11.22696567 & 0.00000000 \\ \mathrm{C} & 0.39883667 & 9.83572079 & 0.00000000 \\ \mathrm{C} & 1.26257300 & 8.58295065 & 0.00000000 \\ \mathrm{C} & 0.12382879 & 7.57380620 & 0.00000000 \\ \mathrm{C} & 0.16012925 & 6.13181423 & 0.00000000\end{array}$




\begin{tabular}{|c|c|c|c|}
\hline $\mathrm{N}$ & -0.88014257 & 9.54609219 & 0.00000000 \\
\hline $\mathrm{C}$ & 2.11981953 & 8.47920873 & 1.28539433 \\
\hline $\mathrm{H}$ & 2.86476662 & 9.27913407 & 1.32076349 \\
\hline $\mathrm{H}$ & 2.64871237 & 7.52277187 & 1.32396030 \\
\hline $\mathrm{H}$ & 1.49388325 & 8.55782463 & 2.17983240 \\
\hline $\mathrm{C}$ & 2.11981953 & 8.47920873 & -1.28539433 \\
\hline $\mathrm{H}$ & 1.49388325 & 8.55782463 & -2.17983240 \\
\hline $\mathrm{H}$ & 2.64871237 & 7.52277187 & -1.32396030 \\
\hline $\mathrm{H}$ & 2.86476662 & 9.27913407 & -1.32076349 \\
\hline $\mathrm{N}$ & -1.04831400 & 8.16149441 & 0.00000000 \\
\hline $\mathrm{C}$ & 0.63185945 & 0.24890642 & 0.00000000 \\
\hline $\mathrm{C}$ & 1.01697331 & 1.63888573 & 0.00000000 \\
\hline $\mathrm{C}$ & 0.15686247 & 2.89398220 & 0.00000000 \\
\hline $\mathrm{C}$ & 1.29859057 & 3.89992648 & 0.00000000 \\
\hline $\mathrm{C}$ & 1.26494119 & 5.34194781 & 0.00000000 \\
\hline $\mathrm{N}$ & 2.29678540 & 1.92484571 & 0.00000000 \\
\hline $\mathrm{C}$ & -0.69992446 & 3.00007492 & -1.28556600 \\
\hline $\mathrm{H}$ & -1.44699564 & 2.20215742 & -1.32121117 \\
\hline $\mathrm{H}$ & -1.22633013 & 3.95783347 & -1.32426499 \\
\hline $\mathrm{H}$ & -0.07398163 & 2.91990272 & -2.17986667 \\
\hline $\mathrm{C}$ & -0.69992446 & 3.00007492 & 1.28556600 \\
\hline $\mathrm{H}$ & -0.07398163 & 2.91990272 & 2.17986667 \\
\hline $\mathrm{H}$ & -1.22633013 & 3.95783347 & 1.32426499 \\
\hline $\mathrm{H}$ & -1.44699564 & 2.20215742 & 1.32121117 \\
\hline $\mathrm{N}$ & 2.46919546 & 3.30874060 & 0.00000000 \\
\hline $\mathrm{H}$ & 2.25757194 & 5.78211448 & 0.00000000 \\
\hline $\mathrm{H}$ & -0.83214551 & 5.69089797 & 0.00000000 \\
\hline $\mathrm{C}$ & 2.33162030 & 22.71287417 & 0.00000000 \\
\hline $\mathrm{H}$ & 1.49636455 & 23.41669674 & 0.00000000 \\
\hline $\mathrm{H}$ & 2.95613026 & 22.90547781 & -0.88186289 \\
\hline $\mathrm{H}$ & 2.95613026 & 22.90547781 & 0.88186289 \\
\hline $\mathrm{C}$ & 1.80863108 & 21.31400322 & 0.00000000 \\
\hline $\mathrm{C}$ & 2.65863588 & 20.05603470 & 0.00000000 \\
\hline
\end{tabular}




\begin{tabular}{|c|c|c|c|}
\hline $\mathrm{C}$ & 1.51255181 & 19.05707760 & 0.00000000 \\
\hline $\mathrm{C}$ & 1.54491586 & 17.61051660 & 0.00000000 \\
\hline $\mathrm{N}$ & 0.54074830 & 21.05926269 & 0.00000000 \\
\hline $\mathrm{C}$ & 3.52629086 & 19.97504417 & 1.27767253 \\
\hline $\mathrm{H}$ & 4.25556480 & 20.79245686 & 1.29158529 \\
\hline $\mathrm{H}$ & 4.07892829 & 19.03196724 & 1.31969771 \\
\hline $\mathrm{H}$ & 2.90977825 & 20.04830125 & 2.17931399 \\
\hline $\mathrm{C}$ & 3.52629086 & 19.97504417 & -1.27767253 \\
\hline $\mathrm{H}$ & 2.90977825 & 20.04830125 & -2.17931399 \\
\hline $\mathrm{H}$ & 4.07892829 & 19.03196724 & -1.31969771 \\
\hline $\mathrm{H}$ & 4.25556480 & 20.79245686 & -1.29158529 \\
\hline $\mathrm{N}$ & 0.35150361 & 19.65120140 & 0.00000000 \\
\hline $\mathrm{C}$ & 2.04196887 & 11.72871127 & 0.00000000 \\
\hline $\mathrm{C}$ & 2.42218484 & 13.12067699 & 0.00000000 \\
\hline $\mathrm{C}$ & 1.55702128 & 14.37220103 & 0.00000000 \\
\hline $\mathrm{C}$ & 2.69443956 & 15.38304933 & 0.00000000 \\
\hline $\mathrm{C}$ & 2.65130269 & 16.82648521 & 0.00000000 \\
\hline $\mathrm{N}$ & 3.70004282 & 13.41149106 & 0.00000000 \\
\hline $\mathrm{C}$ & 0.69992446 & 14.47529418 & -1.28549737 \\
\hline $\mathrm{H}$ & -0.04516288 & 13.67545547 & -1.32085086 \\
\hline $\mathrm{H}$ & 0.17136979 & 15.43180428 & -1.32468767 \\
\hline $\mathrm{H}$ & 1.32618594 & 14.39628563 & -2.17970216 \\
\hline $\mathrm{C}$ & 0.69992446 & 14.47529418 & 1.28549737 \\
\hline $\mathrm{H}$ & 1.32618594 & 14.39628563 & 2.17970216 \\
\hline $\mathrm{H}$ & 0.17136979 & 15.43180428 & 1.32468767 \\
\hline $\mathrm{H}$ & -0.04516288 & 13.67545547 & 1.32085086 \\
\hline $\mathrm{N}$ & 3.86707594 & 14.79797567 & 0.00000000 \\
\hline $\mathrm{H}$ & 3.64119783 & 17.27315822 & 0.00000000 \\
\hline $\mathrm{H}$ & 0.55277771 & 17.16917018 & 0.00000000 \\
\hline $\mathrm{H}$ & -0.07739297 & 11.89284517 & 0.00000000 \\
\hline $\mathrm{H}$ & 2.89994406 & 11.06330501 & 0.00000000 \\
\hline $\mathrm{C}$ & -2.04196887 & -11.72871127 & 0.00000000 \\
\hline $\mathrm{C}$ & -2.42218484 & -13.12067699 & 0.00000000 \\
\hline
\end{tabular}




\begin{tabular}{|c|c|c|c|}
\hline $\mathrm{C}$ & -1.55702128 & -14.37220103 & 0.00000000 \\
\hline $\mathrm{C}$ & -2.69443956 & -15.38304933 & 0.00000000 \\
\hline $\mathrm{C}$ & -2.65130269 & -16.82648521 & 0.00000000 \\
\hline $\mathrm{N}$ & -3.70004282 & -13.41149106 & 0.00000000 \\
\hline $\mathrm{C}$ & -0.69992446 & -14.47529418 & 1.28549737 \\
\hline $\mathrm{H}$ & 0.04516288 & -13.67545547 & 1.32085086 \\
\hline $\mathrm{H}$ & -0.17136979 & -15.43180428 & 1.32468767 \\
\hline $\mathrm{H}$ & -1.32618594 & -14.39628563 & 2.17970216 \\
\hline $\mathrm{C}$ & -0.69992446 & -14.47529418 & -1.28549737 \\
\hline $\mathrm{H}$ & -1.32618594 & -14.39628563 & -2.17970216 \\
\hline $\mathrm{H}$ & -0.17136979 & -15.43180428 & -1.32468767 \\
\hline $\mathrm{H}$ & 0.04516288 & -13.67545547 & -1.32085086 \\
\hline $\mathrm{N}$ & -3.86707594 & -14.79797567 & 0.00000000 \\
\hline $\mathrm{C}$ & -2.33162030 & -22.71287417 & 0.00000000 \\
\hline $\mathrm{H}$ & -1.49636455 & -23.41669674 & 0.00000000 \\
\hline $\mathrm{H}$ & -2.95613026 & -22.90547781 & 0.88186289 \\
\hline $\mathrm{H}$ & -2.95613026 & -22.90547781 & -0.88186289 \\
\hline $\mathrm{C}$ & -1.80863108 & -21.31400322 & 0.00000000 \\
\hline $\mathrm{C}$ & -2.65863588 & -20.05603470 & 0.00000000 \\
\hline $\mathrm{C}$ & -1.51255181 & -19.05707760 & 0.00000000 \\
\hline $\mathrm{C}$ & -1.54491586 & -17.61051660 & 0.00000000 \\
\hline $\mathrm{N}$ & -0.54074830 & -21.05926269 & 0.00000000 \\
\hline $\mathrm{C}$ & -3.52629086 & -19.97504417 & -1.27767253 \\
\hline $\mathrm{H}$ & -4.25556480 & -20.79245686 & -1.29158529 \\
\hline $\mathrm{H}$ & -4.07892829 & -19.03196724 & -1.31969771 \\
\hline $\mathrm{H}$ & -2.90977825 & -20.04830125 & -2.17931399 \\
\hline $\mathrm{C}$ & -3.52629086 & -19.97504417 & 1.27767253 \\
\hline $\mathrm{H}$ & -2.90977825 & -20.04830125 & 2.17931399 \\
\hline $\mathrm{H}$ & -4.07892829 & -19.03196724 & 1.31969771 \\
\hline $\mathrm{H}$ & -4.25556480 & -20.79245686 & 1.29158529 \\
\hline $\mathrm{N}$ & -0.35150361 & -19.65120140 & 0.00000000 \\
\hline $\mathrm{H}$ & -0.55277771 & -17.16917018 & 0.00000000 \\
\hline $\mathrm{H}$ & -3.64119783 & -17.27315822 & 0.00000000 \\
\hline
\end{tabular}




\begin{tabular}{|c|c|c|c|}
\hline $\mathrm{C}$ & -0.63185945 & -0.24890642 & 0.00000000 \\
\hline $\mathrm{C}$ & -1.01697331 & -1.63888573 & 0.00000000 \\
\hline $\mathrm{C}$ & -0.15686247 & -2.89398220 & 0.00000000 \\
\hline $\mathrm{C}$ & -1.29859057 & -3.89992648 & 0.00000000 \\
\hline $\mathrm{C}$ & -1.26494119 & -5.34194781 & 0.00000000 \\
\hline $\mathrm{N}$ & -2.29678540 & -1.92484571 & 0.00000000 \\
\hline $\mathrm{C}$ & 0.69992446 & -3.00007492 & 1.28556600 \\
\hline $\mathrm{H}$ & 1.44699564 & -2.20215742 & 1.32121117 \\
\hline $\mathrm{H}$ & 1.22633013 & -3.95783347 & 1.32426499 \\
\hline $\mathrm{H}$ & 0.07398163 & -2.91990272 & 2.17986667 \\
\hline $\mathrm{C}$ & 0.69992446 & -3.00007492 & -1.28556600 \\
\hline $\mathrm{H}$ & 0.07398163 & -2.91990272 & -2.17986667 \\
\hline $\mathrm{H}$ & 1.22633013 & -3.95783347 & -1.32426499 \\
\hline $\mathrm{H}$ & 1.44699564 & -2.20215742 & -1.32121117 \\
\hline $\mathrm{N}$ & -2.46919546 & -3.30874060 & 0.00000000 \\
\hline $\mathrm{C}$ & -0.78011576 & -11.22696567 & 0.00000000 \\
\hline $\mathrm{C}$ & -0.39883667 & -9.83572079 & 0.00000000 \\
\hline $\mathrm{C}$ & -1.26257300 & -8.58295065 & 0.00000000 \\
\hline $\mathrm{C}$ & -0.12382879 & -7.57380620 & 0.00000000 \\
\hline $\mathrm{C}$ & -0.16012925 & -6.13181423 & 0.00000000 \\
\hline $\mathrm{N}$ & 0.88014257 & -9.54609219 & 0.00000000 \\
\hline $\mathrm{C}$ & -2.11981953 & -8.47920873 & -1.28539433 \\
\hline $\mathrm{H}$ & -2.86476662 & -9.27913407 & -1.32076349 \\
\hline $\mathrm{H}$ & -2.64871237 & -7.52277187 & -1.32396030 \\
\hline $\mathrm{H}$ & -1.49388325 & -8.55782463 & -2.17983240 \\
\hline $\mathrm{C}$ & -2.11981953 & -8.47920873 & 1.28539433 \\
\hline $\mathrm{H}$ & -1.49388325 & -8.55782463 & 2.17983240 \\
\hline $\mathrm{H}$ & -2.64871237 & -7.52277187 & 1.32396030 \\
\hline $\mathrm{H}$ & -2.86476662 & -9.27913407 & 1.32076349 \\
\hline $\mathrm{N}$ & 1.04831400 & -8.16149441 & 0.00000000 \\
\hline $\mathrm{H}$ & 0.83214551 & -5.69089797 & 0.00000000 \\
\hline $\mathrm{H}$ & -2.25757194 & -5.78211448 & 0.00000000 \\
\hline $\mathrm{H}$ & -2.89994406 & -11.06330501 & 0.00000000 \\
\hline
\end{tabular}




$\begin{array}{lrrr}\mathrm{H} & 0.07739297 & -11.89284517 & 0.00000000 \\ \mathrm{H} & 1.48742933 & -0.41953489 & 0.00000000 \\ \mathrm{H} & -1.48742933 & 0.41953489 & 0.00000000\end{array}$

Conjugated polyimine $12 \mathrm{mer}$

Total Energy $=-4574.54926065$ (a.u.)

$\begin{array}{lrrr}\mathrm{C} & 0.57993580 & 0.27522375 & 0.00000000 \\ \mathrm{C} & 0.93758838 & 1.67452000 & 0.00000000 \\ \mathrm{C} & -0.05127698 & 2.82305496 & 0.00000000 \\ \mathrm{C} & 0.96249138 & 3.94930434 & 0.00000000 \\ \mathrm{C} & 0.63396395 & 5.35741352 & 0.00000000 \\ \mathrm{~N} & 2.17565986 & 2.09774386 & 0.00000000 \\ \mathrm{C} & -0.92463681 & 2.85015408 & 1.38887209 \\ \mathrm{H} & -1.57850928 & 1.97200677 & 1.42261676 \\ \mathrm{H} & -1.55852268 & 3.74339928 & 1.39882385 \\ \mathrm{H} & -0.30838567 & 2.85532815 & 2.29328652 \\ \mathrm{C} & -0.92463681 & 2.85015408 & -1.38887209 \\ \mathrm{H} & -0.30838567 & 2.85532815 & -2.29328652 \\ \mathrm{H} & -1.55852268 & 3.74339928 & -1.39882385 \\ \mathrm{H} & -1.57850928 & 1.97200677 & -1.42261676 \\ \mathrm{~N} & 2.19073375 & 3.49922924 & 0.00000000 \\ \mathrm{C} & -0.57470445 & 10.97372361 & 0.00000000 \\ \mathrm{C} & -0.93285433 & 9.52411188 & 0.00000000 \\ \mathrm{C} & 0.05343570 & 8.37371359 & 0.00000000 \\ \mathrm{C} & -0.96524529 & 7.24929631 & 0.00000000 \\ \mathrm{C} & -0.63283683 & 5.83807757 & 0.00000000 \\ \mathrm{~N} & -2.16194524 & 9.12156079 & 0.00000000 \\ \mathrm{C} & 0.92571159 & 8.34385573 & -1.23248824 \\ \mathrm{H} & 1.58829301 & 9.21614682 & -1.26219411 \\ \mathrm{H} & 1.55252827 & 7.44576805 & -1.24843562 \\ \mathrm{H} & 0.30934622 & 8.34685825 & -2.13715793 \\ \mathrm{H} & 0.92571159 & 8.34385573 & 1.23248824 \\ \mathrm{H} & & & \\ \mathrm{H} & & \end{array}$




\begin{tabular}{|c|c|c|c|}
\hline $\mathrm{H}$ & 0.30934622 & 8.34685825 & 2.13715793 \\
\hline $\mathrm{H}$ & 1.55252827 & 7.44576805 & 1.24843562 \\
\hline $\mathrm{H}$ & 1.58829301 & 9.21614682 & 1.26219411 \\
\hline $\mathrm{N}$ & -2.18703073 & 7.69689861 & 0.00000000 \\
\hline $\mathrm{H}$ & -1.45470047 & 5.12590334 & 0.00000000 \\
\hline $\mathrm{H}$ & 1.45655144 & 6.06862254 & 0.00000000 \\
\hline $\mathrm{C}$ & 0.57470445 & -10.97372361 & 0.00000000 \\
\hline $\mathrm{C}$ & 0.93285433 & -9.52411188 & 0.00000000 \\
\hline $\mathrm{C}$ & -0.05343570 & -8.37371359 & 0.00000000 \\
\hline $\mathrm{C}$ & 0.96524529 & -7.24929631 & 0.00000000 \\
\hline $\mathrm{C}$ & 0.63283683 & -5.83807757 & 0.00000000 \\
\hline $\mathrm{N}$ & 2.16194524 & -9.12156079 & 0.00000000 \\
\hline $\mathrm{C}$ & -0.92571159 & -8.34385573 & 1.23248824 \\
\hline $\mathrm{H}$ & -1.58829301 & -9.21614682 & 1.26219411 \\
\hline $\mathrm{H}$ & -1.55252827 & -7.44576805 & 1.24843562 \\
\hline $\mathrm{H}$ & -0.30934622 & -8.34685825 & 2.13715793 \\
\hline $\mathrm{C}$ & -0.92571159 & -8.34385573 & -1.23248824 \\
\hline $\mathrm{H}$ & -0.30934622 & -8.34685825 & -2.13715793 \\
\hline $\mathrm{H}$ & -1.55252827 & -7.44576805 & -1.24843562 \\
\hline $\mathrm{H}$ & -1.58829301 & -9.21614682 & -1.26219411 \\
\hline $\mathrm{N}$ & 2.18703073 & -7.69689861 & 0.00000000 \\
\hline $\mathrm{C}$ & -0.57993580 & -0.27522375 & 0.00000000 \\
\hline $\mathrm{C}$ & -0.93758838 & -1.67452000 & 0.00000000 \\
\hline $\mathrm{C}$ & 0.05127698 & -2.82305496 & 0.00000000 \\
\hline $\mathrm{C}$ & -0.96249138 & -3.94930434 & 0.00000000 \\
\hline $\mathrm{C}$ & -0.63396395 & -5.35741352 & 0.00000000 \\
\hline $\mathrm{N}$ & -2.17565986 & -2.09774386 & 0.00000000 \\
\hline $\mathrm{C}$ & 0.92463681 & -2.85015408 & -1.38887209 \\
\hline $\mathrm{H}$ & 1.57850928 & -1.97200677 & -1.42261676 \\
\hline $\mathrm{H}$ & 1.55852268 & -3.74339928 & -1.39882385 \\
\hline $\mathrm{H}$ & 0.30838567 & -2.85532815 & -2.29328652 \\
\hline $\mathrm{C}$ & 0.92463681 & -2.85015408 & 1.38887209 \\
\hline $\mathrm{H}$ & 0.30838567 & -2.85532815 & 2.29328652 \\
\hline
\end{tabular}




\begin{tabular}{|c|c|c|c|}
\hline $\mathrm{H}$ & 1.55852268 & -3.74339928 & 1.39882385 \\
\hline $\mathrm{H}$ & 1.57850928 & -1.97200677 & 1.42261676 \\
\hline $\mathrm{N}$ & -2.19073375 & -3.49922924 & 0.00000000 \\
\hline $\mathrm{H}$ & -1.45655144 & -6.06862254 & 0.00000000 \\
\hline $\mathrm{H}$ & 1.45470047 & -5.12590334 & 0.00000000 \\
\hline $\mathrm{H}$ & 1.38694429 & -0.45341512 & 0.00000000 \\
\hline $\mathrm{H}$ & -1.38694429 & 0.45341512 & 0.00000000 \\
\hline $\mathrm{C}$ & 0.71987992 & 22.85920713 & 0.00000000 \\
\hline $\mathrm{C}$ & 1.07753250 & 24.25850337 & 0.00000000 \\
\hline $\mathrm{C}$ & 0.08866714 & 25.40703835 & 0.00000000 \\
\hline $\mathrm{C}$ & 1.10243550 & 26.53328771 & 0.00000000 \\
\hline $\mathrm{C}$ & 0.77390806 & 27.94139691 & 0.00000000 \\
\hline $\mathrm{N}$ & 2.31560398 & 24.68172724 & 0.00000000 \\
\hline $\mathrm{C}$ & -0.78479376 & 25.43401294 & 1.17732071 \\
\hline $\mathrm{H}$ & -1.43866624 & 24.55586563 & 1.21106537 \\
\hline $\mathrm{H}$ & -1.41867964 & 26.32725816 & 1.18727247 \\
\hline $\mathrm{H}$ & -0.16854262 & 25.43918702 & 2.08173513 \\
\hline $\mathrm{C}$ & -0.78479376 & 25.43401294 & -1.17732071 \\
\hline $\mathrm{H}$ & -0.16854262 & 25.43918702 & -2.08173513 \\
\hline $\mathrm{H}$ & -1.41867964 & 26.32725816 & -1.18727247 \\
\hline $\mathrm{H}$ & -1.43866624 & 24.55586563 & -1.21106537 \\
\hline $\mathrm{N}$ & 2.33067787 & 26.08321262 & 0.00000000 \\
\hline $\mathrm{C}$ & -0.43476033 & 33.55770699 & 0.00000000 \\
\hline $\mathrm{H}$ & -1.34410678 & 34.16301438 & 0.00000000 \\
\hline $\mathrm{H}$ & 0.16762002 & 33.83097652 & 0.67406190 \\
\hline $\mathrm{H}$ & 0.16762002 & 33.83097652 & -0.67406190 \\
\hline $\mathrm{C}$ & -0.79291020 & 32.10809525 & 0.00000000 \\
\hline $\mathrm{C}$ & 0.19337984 & 30.95769698 & 0.00000000 \\
\hline $\mathrm{C}$ & -0.82530117 & 29.83327970 & 0.00000000 \\
\hline $\mathrm{C}$ & -0.49289271 & 28.42206096 & 0.00000000 \\
\hline $\mathrm{N}$ & -2.02200111 & 31.70554417 & 0.00000000 \\
\hline $\mathrm{C}$ & 1.06555463 & 30.92771461 & -1.44403962 \\
\hline $\mathrm{H}$ & 1.72813607 & 31.80000571 & -1.47374550 \\
\hline
\end{tabular}




\begin{tabular}{|c|c|c|c|}
\hline $\mathrm{H}$ & 1.69237132 & 30.02962692 & -1.45998700 \\
\hline $\mathrm{H}$ & 0.44918927 & 30.93071713 & -2.34870933 \\
\hline $\mathrm{C}$ & 1.06555463 & 30.92771461 & 1.44403962 \\
\hline $\mathrm{H}$ & 0.44918927 & 30.93071713 & 2.34870933 \\
\hline $\mathrm{H}$ & 1.69237132 & 30.02962692 & 1.45998700 \\
\hline $\mathrm{H}$ & 1.72813607 & 31.80000571 & 1.47374550 \\
\hline $\mathrm{N}$ & -2.04708659 & 30.28088201 & 0.00000000 \\
\hline $\mathrm{H}$ & -1.31475636 & 27.70988672 & 0.00000000 \\
\hline $\mathrm{H}$ & 1.59649558 & 28.65260592 & 0.00000000 \\
\hline $\mathrm{C}$ & 0.59617264 & 11.70505551 & 0.00000000 \\
\hline $\mathrm{C}$ & 0.95432253 & 13.15466723 & 0.00000000 \\
\hline $\mathrm{C}$ & -0.03196750 & 14.30506553 & 0.00000000 \\
\hline $\mathrm{C}$ & 0.98671349 & 15.42948279 & 0.00000000 \\
\hline $\mathrm{C}$ & 0.65430502 & 16.84070154 & 0.00000000 \\
\hline $\mathrm{N}$ & 2.18341343 & 13.55721832 & 0.00000000 \\
\hline $\mathrm{C}$ & -0.90424340 & 14.33492338 & 1.33221032 \\
\hline $\mathrm{H}$ & -1.56682481 & 13.46263229 & 1.36191620 \\
\hline $\mathrm{H}$ & -1.53106007 & 15.23301107 & 1.34815771 \\
\hline $\mathrm{H}$ & -0.28787802 & 14.33192087 & 2.23688003 \\
\hline $\mathrm{C}$ & -0.90424340 & 14.33492338 & -1.33221032 \\
\hline $\mathrm{H}$ & -0.28787802 & 14.33192087 & -2.23688003 \\
\hline $\mathrm{H}$ & -1.53106007 & 15.23301107 & -1.34815771 \\
\hline $\mathrm{H}$ & -1.56682481 & 13.46263229 & -1.36191620 \\
\hline $\mathrm{N}$ & 2.20849893 & 14.98188050 & 0.00000000 \\
\hline $\mathrm{C}$ & -0.55846761 & 22.40355536 & 0.00000000 \\
\hline $\mathrm{C}$ & -0.91612018 & 21.00425912 & 0.00000000 \\
\hline $\mathrm{C}$ & 0.07274518 & 19.85572415 & 0.00000000 \\
\hline $\mathrm{C}$ & -0.94102318 & 18.72947478 & 0.00000000 \\
\hline $\mathrm{C}$ & -0.61249575 & 17.32136559 & 0.00000000 \\
\hline $\mathrm{N}$ & -2.15419166 & 20.58103524 & 0.00000000 \\
\hline $\mathrm{C}$ & 0.94610501 & 19.82862503 & -1.28915000 \\
\hline $\mathrm{H}$ & 1.59997748 & 20.70677233 & -1.32289467 \\
\hline $\mathrm{H}$ & 1.57999088 & 18.93537982 & -1.29910176 \\
\hline
\end{tabular}




\begin{tabular}{|c|c|c|c|}
\hline $\mathrm{H}$ & 0.32985387 & 19.82345096 & -2.19356443 \\
\hline $\mathrm{C}$ & 0.94610501 & 19.82862503 & 1.28915000 \\
\hline $\mathrm{H}$ & 0.32985387 & 19.82345096 & 2.19356443 \\
\hline $\mathrm{H}$ & 1.57999088 & 18.93537982 & 1.29910176 \\
\hline $\mathrm{H}$ & 1.59997748 & 20.70677233 & 1.32289467 \\
\hline $\mathrm{N}$ & -2.16926555 & 19.17954987 & 0.00000000 \\
\hline $\mathrm{H}$ & -1.43508324 & 16.61015657 & 0.00000000 \\
\hline $\mathrm{H}$ & 1.47616866 & 17.55287577 & 0.00000000 \\
\hline $\mathrm{H}$ & 1.52688841 & 22.13056827 & 0.00000000 \\
\hline $\mathrm{H}$ & -1.36547609 & 23.13219423 & 0.00000000 \\
\hline $\mathrm{H}$ & -1.42663130 & 11.61896026 & 0.00000000 \\
\hline $\mathrm{H}$ & 1.44809949 & 11.05981885 & 0.00000000 \\
\hline $\mathrm{C}$ & 0.55846761 & -22.40355536 & 0.00000000 \\
\hline $\mathrm{C}$ & 0.91612018 & -21.00425912 & 0.00000000 \\
\hline $\mathrm{C}$ & -0.07274518 & -19.85572415 & 0.00000000 \\
\hline $\mathrm{C}$ & 0.94102318 & -18.72947478 & 0.00000000 \\
\hline $\mathrm{C}$ & 0.61249575 & -17.32136559 & 0.00000000 \\
\hline $\mathrm{N}$ & 2.15419166 & -20.58103524 & 0.00000000 \\
\hline $\mathrm{C}$ & -0.94610501 & -19.82862503 & 1.28915000 \\
\hline $\mathrm{H}$ & -1.59997748 & -20.70677233 & 1.32289467 \\
\hline $\mathrm{H}$ & -1.57999088 & -18.93537982 & 1.29910176 \\
\hline $\mathrm{H}$ & -0.32985387 & -19.82345096 & 2.19356443 \\
\hline $\mathrm{C}$ & -0.94610501 & -19.82862503 & -1.28915000 \\
\hline $\mathrm{H}$ & -0.32985387 & -19.82345096 & -2.19356443 \\
\hline $\mathrm{H}$ & -1.57999088 & -18.93537982 & -1.29910176 \\
\hline $\mathrm{H}$ & -1.59997748 & -20.70677233 & -1.32289467 \\
\hline $\mathrm{N}$ & 2.16926555 & -19.17954987 & 0.00000000 \\
\hline $\mathrm{C}$ & -0.59617264 & -11.70505551 & 0.00000000 \\
\hline $\mathrm{C}$ & -0.95432253 & -13.15466723 & 0.00000000 \\
\hline $\mathrm{C}$ & 0.03196750 & -14.30506553 & 0.00000000 \\
\hline $\mathrm{C}$ & -0.98671349 & -15.42948279 & 0.00000000 \\
\hline $\mathrm{C}$ & -0.65430502 & -16.84070154 & 0.00000000 \\
\hline $\mathrm{N}$ & -2.18341343 & -13.55721832 & 0.00000000 \\
\hline
\end{tabular}




\begin{tabular}{|c|c|c|c|}
\hline $\mathrm{C}$ & 0.90424340 & -14.33492338 & -1.33221032 \\
\hline $\mathrm{H}$ & 1.56682481 & -13.46263229 & -1.36191620 \\
\hline $\mathrm{H}$ & 1.53106007 & -15.23301107 & -1.34815771 \\
\hline $\mathrm{H}$ & 0.28787802 & -14.33192087 & -2.23688003 \\
\hline $\mathrm{C}$ & 0.90424340 & -14.33492338 & 1.33221032 \\
\hline $\mathrm{H}$ & 0.28787802 & -14.33192087 & 2.23688003 \\
\hline $\mathrm{H}$ & 1.53106007 & -15.23301107 & 1.34815771 \\
\hline $\mathrm{H}$ & 1.56682481 & -13.46263229 & 1.36191620 \\
\hline $\mathrm{N}$ & -2.20849893 & -14.98188050 & 0.00000000 \\
\hline $\mathrm{H}$ & -1.47616866 & -17.55287577 & 0.00000000 \\
\hline $\mathrm{H}$ & 1.43508324 & -16.61015657 & 0.00000000 \\
\hline $\mathrm{C}$ & 0.43476033 & -33.55770699 & 0.00000000 \\
\hline $\mathrm{C}$ & 0.79291020 & -32.10809525 & 0.00000000 \\
\hline $\mathrm{C}$ & -0.19337984 & -30.95769698 & 0.00000000 \\
\hline $\mathrm{C}$ & 0.82530117 & -29.83327970 & 0.00000000 \\
\hline $\mathrm{C}$ & 0.49289271 & -28.42206096 & 0.00000000 \\
\hline $\mathrm{N}$ & 2.02200111 & -31.70554417 & 0.00000000 \\
\hline $\mathrm{C}$ & -1.06555463 & -30.92771461 & 1.44403962 \\
\hline $\mathrm{H}$ & -1.72813607 & -31.80000571 & 1.47374550 \\
\hline $\mathrm{H}$ & -1.69237132 & -30.02962692 & 1.45998700 \\
\hline $\mathrm{H}$ & -0.44918927 & -30.93071713 & 2.34870933 \\
\hline $\mathrm{C}$ & -1.06555463 & -30.92771461 & -1.44403962 \\
\hline $\mathrm{H}$ & -0.44918927 & -30.93071713 & -2.34870933 \\
\hline $\mathrm{H}$ & -1.69237132 & -30.02962692 & -1.45998700 \\
\hline $\mathrm{H}$ & -1.72813607 & -31.80000571 & -1.47374550 \\
\hline $\mathrm{N}$ & 2.04708659 & -30.28088201 & 0.00000000 \\
\hline $\mathrm{C}$ & -0.71987992 & -22.85920713 & 0.00000000 \\
\hline $\mathrm{C}$ & -1.07753250 & -24.25850337 & 0.00000000 \\
\hline $\mathrm{C}$ & -0.08866714 & -25.40703835 & 0.00000000 \\
\hline $\mathrm{C}$ & -1.10243550 & -26.53328771 & 0.00000000 \\
\hline $\mathrm{C}$ & -0.77390806 & -27.94139691 & 0.00000000 \\
\hline $\mathrm{N}$ & -2.31560398 & -24.68172724 & 0.00000000 \\
\hline $\mathrm{C}$ & 0.78479376 & -25.43401294 & -1.177320 \\
\hline
\end{tabular}




$\begin{array}{lrrr}\mathrm{H} & 1.43866624 & -24.55586563 & -1.21106537 \\ \mathrm{H} & 1.41867964 & -26.32725816 & -1.18727247 \\ \mathrm{H} & 0.16854262 & -25.43918702 & -2.08173513 \\ \mathrm{C} & 0.78479376 & -25.43401294 & 1.17732071 \\ \mathrm{H} & 0.16854262 & -25.43918702 & 2.08173513 \\ \mathrm{H} & 1.41867964 & -26.32725816 & 1.18727247 \\ \mathrm{H} & 1.43866624 & -24.55586563 & 1.21106537 \\ \mathrm{~N} & -2.33067787 & -26.08321262 & 0.00000000 \\ \mathrm{H} & -1.59649558 & -28.65260592 & 0.00000000 \\ \mathrm{H} & 1.31475636 & -27.70988672 & 0.00000000 \\ \mathrm{H} & 1.36547609 & -23.13219423 & 0.00000000 \\ \mathrm{H} & -1.52688841 & -22.13056827 & 0.00000000 \\ \mathrm{H} & 1.34410678 & -34.16301438 & 0.00000000 \\ \mathrm{H} & 1.42663130 & -11.61896026 & 0.00000000 \\ \mathrm{H} & -1.44809949 & -11.05981885 & 0.00000000 \\ \mathrm{H} & -0.16762002 & -33.83097652 & 0.67406190 \\ \mathrm{H} & -0.16762002 & -33.83097652 & -0.67406190\end{array}$

Conjugated polyimine $16 \mathrm{mer}$

Total Energy $=-6098.68557789$ (a.u.)

$\begin{array}{rrrr}\mathrm{C} & 0.58876678 & 11.88714461 & 0.00000000 \\ \mathrm{C} & 0.79963816 & 13.31403270 & 0.00000000 \\ \mathrm{C} & -0.20911028 & 14.45361294 & 0.00000000 \\ \mathrm{C} & 0.80059003 & 15.59239693 & 0.00000000 \\ \mathrm{C} & 0.59075386 & 17.01943139 & 0.00000000 \\ \mathrm{~N} & 2.03446156 & 13.75549003 & 0.00000000 \\ \mathrm{C} & -1.07205235 & 14.45395558 & 1.28534960 \\ \mathrm{H} & -1.71409966 & 13.56938713 & 1.32207700 \\ \mathrm{H} & -1.71329140 & 15.33909608 & 1.32213167 \\ \mathrm{H} & -0.44106385 & 14.45365575 & 2.17967283 \\ \mathrm{C} & -1.07205235 & 14.45395558 & -1.28534960 \\ \mathrm{H} & -0.44106385 & 14.45365575 & -2.17967283 \\ & & & \\ & & \mathrm{~S}-38 & \end{array}$




\begin{tabular}{|c|c|c|c|}
\hline $\mathrm{H}$ & -1.71329140 & 15.33909608 & -1.32213167 \\
\hline $\mathrm{H}$ & -1.71409966 & 13.56938713 & -1.32207700 \\
\hline $\mathrm{N}$ & 2.03508685 & 15.14990611 & 0.00000000 \\
\hline $\mathrm{C}$ & -0.59554437 & 22.80175462 & 0.00000000 \\
\hline $\mathrm{C}$ & -0.80844063 & 21.37512816 & 0.00000000 \\
\hline $\mathrm{C}$ & 0.19951475 & 20.23472399 & 0.00000000 \\
\hline $\mathrm{C}$ & -0.81102805 & 19.09672800 & 0.00000000 \\
\hline $\mathrm{C}$ & -0.60186183 & 17.66953717 & 0.00000000 \\
\hline $\mathrm{N}$ & -2.04361280 & 20.93459710 & 0.00000000 \\
\hline $\mathrm{C}$ & 1.06229830 & 20.23365807 & -1.28548107 \\
\hline $\mathrm{H}$ & 1.70510529 & 21.11762919 & -1.32235571 \\
\hline $\mathrm{H}$ & 1.70273193 & 19.34795972 & -1.32246386 \\
\hline $\mathrm{H}$ & 0.43105196 & 20.23457339 & -2.17961913 \\
\hline $\mathrm{C}$ & 1.06229830 & 20.23365807 & 1.28548107 \\
\hline $\mathrm{H}$ & 0.43105196 & 20.23457339 & 2.17961913 \\
\hline $\mathrm{H}$ & 1.70273193 & 19.34795972 & 1.32246386 \\
\hline $\mathrm{H}$ & 1.70510529 & 21.11762919 & 1.32235571 \\
\hline $\mathrm{N}$ & -2.04512085 & 19.54007873 & 0.00000000 \\
\hline $\mathrm{H}$ & -1.53374974 & 17.11212165 & 0.00000000 \\
\hline $\mathrm{H}$ & 1.52250837 & 17.57700407 & 0.00000000 \\
\hline $\mathrm{C}$ & 0.59627786 & 0.32510182 & 0.00000000 \\
\hline $\mathrm{C}$ & 0.80559983 & 1.75222982 & 0.00000000 \\
\hline $\mathrm{C}$ & -0.20461125 & 2.89053688 & 0.00000000 \\
\hline $\mathrm{C}$ & 0.80365105 & 4.03057843 & 0.00000000 \\
\hline $\mathrm{C}$ & 0.59191559 & 5.45735501 & 0.00000000 \\
\hline $\mathrm{N}$ & 2.03987217 & 2.19524269 & 0.00000000 \\
\hline $\mathrm{C}$ & -1.06750598 & 2.88978769 & 1.28540046 \\
\hline $\mathrm{H}$ & -1.70827443 & 2.00431628 & 1.32227229 \\
\hline $\mathrm{H}$ & -1.71001899 & 3.77400228 & 1.32213231 \\
\hline $\mathrm{H}$ & -0.43644194 & 2.89047639 & 2.17966785 \\
\hline $\mathrm{C}$ & -1.06750598 & 2.88978769 & -1.28540046 \\
\hline $\mathrm{H}$ & -0.43644194 & 2.89047639 & -2.17966785 \\
\hline $\mathrm{H}$ & -1.71001899 & 3.77400228 & -1.32213231 \\
\hline
\end{tabular}




\begin{tabular}{|c|c|c|c|}
\hline $\mathrm{H}$ & -1.70827443 & 2.00431628 & -1.32227229 \\
\hline $\mathrm{N}$ & 2.03868788 & 3.58967877 & 0.00000000 \\
\hline $\mathrm{C}$ & -0.60430791 & 11.23790107 & 0.00000000 \\
\hline $\mathrm{C}$ & -0.81438131 & 9.81086714 & 0.00000000 \\
\hline $\mathrm{C}$ & 0.19549659 & 8.67222931 & 0.00000000 \\
\hline $\mathrm{C}$ & -0.81312615 & 7.53250952 & 0.00000000 \\
\hline $\mathrm{C}$ & -0.60166240 & 6.10567160 & 0.00000000 \\
\hline $\mathrm{N}$ & -2.04879212 & 9.36824619 & 0.00000000 \\
\hline $\mathrm{C}$ & 1.05833083 & 8.67269213 & -1.28545157 \\
\hline $\mathrm{H}$ & 1.69941535 & 9.55791909 & -1.32236865 \\
\hline $\mathrm{H}$ & 1.70051317 & 7.78825030 & -1.32226589 \\
\hline $\mathrm{H}$ & 0.42716729 & 8.67225915 & -2.17964734 \\
\hline $\mathrm{C}$ & 1.05833083 & 8.67269213 & 1.28545157 \\
\hline $\mathrm{H}$ & 0.42716729 & 8.67225915 & 2.17964734 \\
\hline $\mathrm{H}$ & 1.70051317 & 7.78825030 & 1.32226589 \\
\hline $\mathrm{H}$ & 1.69941535 & 9.55791909 & 1.32236865 \\
\hline $\mathrm{N}$ & -2.04800490 & 7.97377281 & 0.00000000 \\
\hline $\mathrm{H}$ & -1.53265707 & 5.54680063 & 0.00000000 \\
\hline $\mathrm{H}$ & 1.52286193 & 6.01628181 & 0.00000000 \\
\hline $\mathrm{H}$ & 1.52013672 & 11.32891177 & 0.00000000 \\
\hline $\mathrm{H}$ & -1.53578232 & 11.79599277 & 0.00000000 \\
\hline $\mathrm{C}$ & 0.63977200 & 35.00860347 & 0.00000000 \\
\hline $\mathrm{C}$ & 0.86008201 & 36.43463704 & 0.00000000 \\
\hline $\mathrm{C}$ & -0.14124507 & 37.58069113 & 0.00000000 \\
\hline $\mathrm{C}$ & 0.87571448 & 38.71323628 & 0.00000000 \\
\hline $\mathrm{C}$ & 0.67131141 & 40.14277480 & 0.00000000 \\
\hline $\mathrm{N}$ & 2.09697031 & 36.86762902 & 0.00000000 \\
\hline $\mathrm{C}$ & -1.00416927 & 37.58692151 & 1.28522897 \\
\hline $\mathrm{H}$ & -1.65314454 & 36.70734086 & 1.32148303 \\
\hline $\mathrm{H}$ & -1.63818657 & 38.47716999 & 1.32261936 \\
\hline $\mathrm{H}$ & -0.37305003 & 37.58114199 & 2.17946651 \\
\hline $\mathrm{C}$ & -1.00416927 & 37.58692151 & -1.28522897 \\
\hline $\mathrm{H}$ & -0.37305003 & 37.58114199 & -2.17946651 \\
\hline
\end{tabular}




\begin{tabular}{|c|c|c|c|}
\hline $\mathrm{H}$ & -1.63818657 & 38.47716999 & -1.32261936 \\
\hline $\mathrm{H}$ & -1.65314454 & 36.70734086 & -1.32148303 \\
\hline $\mathrm{N}$ & 2.10673862 & 38.26391495 & 0.00000000 \\
\hline $\mathrm{C}$ & -0.30311165 & 45.95690736 & 0.00000000 \\
\hline $\mathrm{H}$ & -1.21166167 & 46.56321087 & 0.00000000 \\
\hline $\mathrm{H}$ & 0.29605711 & 46.21788943 & 0.88184547 \\
\hline $\mathrm{H}$ & 0.29605711 & 46.21788943 & -0.88184547 \\
\hline $\mathrm{C}$ & -0.66687407 & 44.50838630 & 0.00000000 \\
\hline $\mathrm{C}$ & 0.31839038 & 43.35307526 & 0.00000000 \\
\hline $\mathrm{C}$ & -0.70951051 & 42.23247708 & 0.00000000 \\
\hline $\mathrm{C}$ & -0.51578888 & 40.79855401 & 0.00000000 \\
\hline $\mathrm{N}$ & -1.89844374 & 44.11375909 & 0.00000000 \\
\hline $\mathrm{C}$ & 1.18897467 & 43.36798737 & -1.27768753 \\
\hline $\mathrm{H}$ & 1.82316346 & 44.26119686 & -1.29313296 \\
\hline $\mathrm{H}$ & 1.84243939 & 42.49168741 & -1.31833502 \\
\hline $\mathrm{H}$ & 0.56781400 & 43.37091192 & -2.17911483 \\
\hline $\mathrm{C}$ & 1.18897467 & 43.36798737 & 1.27768753 \\
\hline $\mathrm{H}$ & 0.56781400 & 43.37091192 & 2.17911483 \\
\hline $\mathrm{H}$ & 1.84243939 & 42.49168741 & 1.31833502 \\
\hline $\mathrm{H}$ & 1.82316346 & 44.26119686 & 1.29313296 \\
\hline $\mathrm{N}$ & -1.92955669 & 42.69339401 & 0.00000000 \\
\hline $\mathrm{H}$ & -1.45253870 & 40.24922251 & 0.00000000 \\
\hline $\mathrm{H}$ & 1.60557949 & 40.69662029 & 0.00000000 \\
\hline $\mathrm{C}$ & 0.59897111 & 23.44832249 & 0.00000000 \\
\hline $\mathrm{C}$ & 0.81348718 & 24.87468024 & 0.00000000 \\
\hline $\mathrm{C}$ & -0.19216475 & 26.01696791 & 0.00000000 \\
\hline $\mathrm{C}$ & 0.82059832 & 27.15310871 & 0.00000000 \\
\hline $\mathrm{C}$ & 0.61433334 & 28.58066885 & 0.00000000 \\
\hline $\mathrm{N}$ & 2.04943640 & 25.31281005 & 0.00000000 \\
\hline $\mathrm{C}$ & -1.05511863 & 26.01962141 & 1.28532062 \\
\hline $\mathrm{H}$ & -1.69983883 & 25.13697027 & 1.32184969 \\
\hline $\mathrm{H}$ & -1.69364620 & 26.90669955 & 1.32230121 \\
\hline $\mathrm{H}$ & -0.42412809 & 26.01724567 & 2.17964213 \\
\hline
\end{tabular}




\begin{tabular}{|c|c|c|c|}
\hline $\mathrm{C}$ & -1.05511863 & 26.01962141 & -1.28532062 \\
\hline $\mathrm{H}$ & -0.42412809 & 26.01724567 & -2.17964213 \\
\hline $\mathrm{H}$ & -1.69364620 & 26.90669955 & -1.32230121 \\
\hline $\mathrm{H}$ & -1.69983883 & 25.13697027 & -1.32184969 \\
\hline $\mathrm{N}$ & 2.05392243 & 26.70727243 & 0.00000000 \\
\hline $\mathrm{C}$ & -0.55675842 & 34.36628982 & 0.00000000 \\
\hline $\mathrm{C}$ & -0.77420608 & 32.94014533 & 0.00000000 \\
\hline $\mathrm{C}$ & 0.23094051 & 31.79709559 & 0.00000000 \\
\hline $\mathrm{C}$ & -0.78232290 & 30.66158843 & 0.00000000 \\
\hline $\mathrm{C}$ & -0.57660197 & 29.23382042 & 0.00000000 \\
\hline $\mathrm{N}$ & -2.01041289 & 32.50271408 & 0.00000000 \\
\hline $\mathrm{C}$ & 1.09367094 & 31.79378934 & -1.28546175 \\
\hline $\mathrm{H}$ & 1.73884458 & 32.67603323 & -1.32233834 \\
\hline $\mathrm{H}$ & 1.73164133 & 30.90629798 & -1.32262976 \\
\hline $\mathrm{H}$ & 0.46234335 & 31.79655123 & -2.17954836 \\
\hline $\mathrm{C}$ & 1.09367094 & 31.79378934 & 1.28546175 \\
\hline $\mathrm{H}$ & 0.46234335 & 31.79655123 & 2.17954836 \\
\hline $\mathrm{H}$ & 1.73164133 & 30.90629798 & 1.32262976 \\
\hline $\mathrm{H}$ & 1.73884458 & 32.67603323 & 1.32233834 \\
\hline $\mathrm{N}$ & -2.01515773 & 31.10791642 & 0.00000000 \\
\hline $\mathrm{H}$ & -1.50995546 & 28.67881821 & 0.00000000 \\
\hline $\mathrm{H}$ & 1.54749464 & 29.13590115 & 0.00000000 \\
\hline $\mathrm{H}$ & 1.56775613 & 34.44473352 & 0.00000000 \\
\hline $\mathrm{H}$ & -1.48499848 & 34.92969732 & 0.00000000 \\
\hline $\mathrm{H}$ & -1.52576158 & 23.36192658 & 0.00000000 \\
\hline $\mathrm{H}$ & 1.52899355 & 22.88786229 & 0.00000000 \\
\hline $\mathrm{C}$ & 0.55675842 & -34.36628982 & 0.00000000 \\
\hline $\mathrm{C}$ & 0.77420608 & -32.94014533 & 0.00000000 \\
\hline $\mathrm{C}$ & -0.23094051 & -31.79709559 & 0.00000000 \\
\hline $\mathrm{C}$ & 0.78232290 & -30.66158843 & 0.00000000 \\
\hline $\mathrm{C}$ & 0.57660197 & -29.23382042 & 0.00000000 \\
\hline $\mathrm{N}$ & 2.01041289 & -32.50271408 & 0.00000000 \\
\hline $\mathrm{C}$ & -1.09367094 & -31.79378934 & 1.28546175 \\
\hline
\end{tabular}




\begin{tabular}{|c|c|c|c|}
\hline $\mathrm{H}$ & -1.73884458 & -32.67603323 & 1.32233834 \\
\hline $\mathrm{H}$ & -1.73164133 & -30.90629798 & 1.32262976 \\
\hline $\mathrm{H}$ & -0.46234335 & -31.79655123 & 2.17954836 \\
\hline $\mathrm{C}$ & -1.09367094 & -31.79378934 & -1.28546175 \\
\hline $\mathrm{H}$ & -0.46234335 & -31.79655123 & -2.17954836 \\
\hline $\mathrm{H}$ & -1.73164133 & -30.90629798 & -1.32262976 \\
\hline $\mathrm{H}$ & -1.73884458 & -32.67603323 & -1.32233834 \\
\hline $\mathrm{N}$ & 2.01515773 & -31.10791642 & 0.00000000 \\
\hline $\mathrm{C}$ & -0.59897111 & -23.44832249 & 0.00000000 \\
\hline $\mathrm{C}$ & -0.81348718 & -24.87468024 & 0.00000000 \\
\hline $\mathrm{C}$ & 0.19216475 & -26.01696791 & 0.00000000 \\
\hline $\mathrm{C}$ & -0.82059832 & -27.15310871 & 0.00000000 \\
\hline $\mathrm{C}$ & -0.61433334 & -28.58066885 & 0.00000000 \\
\hline $\mathrm{N}$ & -2.04943640 & -25.31281005 & 0.00000000 \\
\hline $\mathrm{C}$ & 1.05511863 & -26.01962141 & -1.28532062 \\
\hline $\mathrm{H}$ & 1.69983883 & -25.13697027 & -1.32184969 \\
\hline $\mathrm{H}$ & 1.69364620 & -26.90669955 & -1.32230121 \\
\hline $\mathrm{H}$ & 0.42412809 & -26.01724567 & -2.17964213 \\
\hline $\mathrm{C}$ & 1.05511863 & -26.01962141 & 1.28532062 \\
\hline $\mathrm{H}$ & 0.42412809 & -26.01724567 & 2.17964213 \\
\hline $\mathrm{H}$ & 1.69364620 & -26.90669955 & 1.32230121 \\
\hline $\mathrm{H}$ & 1.69983883 & -25.13697027 & 1.32184969 \\
\hline $\mathrm{N}$ & -2.05392243 & -26.70727243 & 0.00000000 \\
\hline $\mathrm{H}$ & -1.54749464 & -29.13590115 & 0.00000000 \\
\hline $\mathrm{H}$ & 1.50995546 & -28.67881821 & 0.00000000 \\
\hline $\mathrm{C}$ & 0.30311165 & -45.95690736 & 0.00000000 \\
\hline $\mathrm{H}$ & 1.21166167 & -46.56321087 & 0.00000000 \\
\hline $\mathrm{H}$ & -0.29605711 & -46.21788943 & -0.88184547 \\
\hline $\mathrm{H}$ & -0.29605711 & -46.21788943 & 0.88184547 \\
\hline $\mathrm{C}$ & 0.66687407 & -44.50838630 & 0.00000000 \\
\hline $\mathrm{C}$ & -0.31839038 & -43.35307526 & 0.00000000 \\
\hline $\mathrm{C}$ & 0.70951051 & -42.23247708 & 0.00000000 \\
\hline $\mathrm{C}$ & 0.51578888 & -40.79855401 & 0.00000000 \\
\hline
\end{tabular}




\begin{tabular}{|c|c|c|c|}
\hline $\mathrm{N}$ & 1.89844374 & -44.11375909 & 0.00000000 \\
\hline $\mathrm{C}$ & -1.18897467 & -43.36798737 & 1.27768753 \\
\hline $\mathrm{H}$ & -1.82316346 & -44.26119686 & 1.29313296 \\
\hline $\mathrm{H}$ & -1.84243939 & -42.49168741 & 1.31833502 \\
\hline $\mathrm{H}$ & -0.56781400 & -43.37091192 & 2.17911483 \\
\hline $\mathrm{C}$ & -1.18897467 & -43.36798737 & -1.27768753 \\
\hline $\mathrm{H}$ & -0.56781400 & -43.37091192 & -2.17911483 \\
\hline $\mathrm{H}$ & -1.84243939 & -42.49168741 & -1.31833502 \\
\hline $\mathrm{H}$ & -1.82316346 & -44.26119686 & -1.29313296 \\
\hline $\mathrm{N}$ & 1.92955669 & -42.69339401 & 0.00000000 \\
\hline $\mathrm{C}$ & -0.63977200 & -35.00860347 & 0.00000000 \\
\hline $\mathrm{C}$ & -0.86008201 & -36.43463704 & 0.00000000 \\
\hline $\mathrm{C}$ & 0.14124507 & -37.58069113 & 0.00000000 \\
\hline $\mathrm{C}$ & -0.87571448 & -38.71323628 & 0.00000000 \\
\hline $\mathrm{C}$ & -0.67131141 & -40.14277480 & 0.00000000 \\
\hline $\mathrm{N}$ & -2.09697031 & -36.86762902 & 0.00000000 \\
\hline $\mathrm{C}$ & 1.00416927 & -37.58692151 & -1.28522897 \\
\hline $\mathrm{H}$ & 1.65314454 & -36.70734086 & -1.32148303 \\
\hline $\mathrm{H}$ & 1.63818657 & -38.47716999 & -1.32261936 \\
\hline $\mathrm{H}$ & 0.37305003 & -37.58114199 & -2.17946651 \\
\hline $\mathrm{C}$ & 1.00416927 & -37.58692151 & 1.28522897 \\
\hline $\mathrm{H}$ & 0.37305003 & -37.58114199 & 2.17946651 \\
\hline $\mathrm{H}$ & 1.63818657 & -38.47716999 & 1.32261936 \\
\hline $\mathrm{H}$ & 1.65314454 & -36.70734086 & 1.32148303 \\
\hline $\mathrm{N}$ & -2.10673862 & -38.26391495 & 0.00000000 \\
\hline $\mathrm{H}$ & -1.60557949 & -40.69662029 & 0.00000000 \\
\hline $\mathrm{H}$ & 1.45253870 & -40.24922251 & 0.00000000 \\
\hline $\mathrm{H}$ & 1.48499848 & -34.92969732 & 0.00000000 \\
\hline $\mathrm{H}$ & -1.56775613 & -34.44473352 & 0.00000000 \\
\hline $\mathrm{C}$ & 0.60430791 & -11.23790107 & 0.00000000 \\
\hline $\mathrm{C}$ & 0.81438131 & -9.81086714 & 0.00000000 \\
\hline $\mathrm{C}$ & -0.19549659 & -8.67222931 & 0.00000000 \\
\hline $\mathrm{C}$ & 0.81312615 & -7.53250952 & 0.00000000 \\
\hline
\end{tabular}




\begin{tabular}{|c|c|c|c|}
\hline $\mathrm{C}$ & 0.60166240 & -6.10567160 & 0.00000000 \\
\hline $\mathrm{N}$ & 2.04879212 & -9.36824619 & 0.00000000 \\
\hline $\mathrm{C}$ & -1.05833083 & -8.67269213 & 1.28545157 \\
\hline $\mathrm{H}$ & -1.69941535 & -9.55791909 & 1.32236865 \\
\hline $\mathrm{H}$ & -1.70051317 & -7.78825030 & 1.32226589 \\
\hline $\mathrm{H}$ & -0.42716729 & -8.67225915 & 2.17964734 \\
\hline $\mathrm{C}$ & -1.05833083 & -8.67269213 & -1.28545157 \\
\hline $\mathrm{H}$ & -0.42716729 & -8.67225915 & -2.17964734 \\
\hline $\mathrm{H}$ & -1.70051317 & -7.78825030 & -1.32226589 \\
\hline $\mathrm{H}$ & -1.69941535 & -9.55791909 & -1.32236865 \\
\hline $\mathrm{N}$ & 2.04800490 & -7.97377281 & 0.00000000 \\
\hline $\mathrm{C}$ & -0.59627786 & -0.32510182 & 0.00000000 \\
\hline $\mathrm{C}$ & -0.80559983 & -1.75222982 & 0.00000000 \\
\hline $\mathrm{C}$ & 0.20461125 & -2.89053688 & 0.00000000 \\
\hline $\mathrm{C}$ & -0.80365105 & -4.03057843 & 0.00000000 \\
\hline $\mathrm{C}$ & -0.59191559 & -5.45735501 & 0.00000000 \\
\hline $\mathrm{N}$ & -2.03987217 & -2.19524269 & 0.00000000 \\
\hline $\mathrm{C}$ & 1.06750598 & -2.88978769 & -1.28540046 \\
\hline $\mathrm{H}$ & 1.70827443 & -2.00431628 & -1.32227229 \\
\hline $\mathrm{H}$ & 1.71001899 & -3.77400228 & -1.32213231 \\
\hline $\mathrm{H}$ & 0.43644194 & -2.89047639 & -2.17966785 \\
\hline $\mathrm{C}$ & 1.06750598 & -2.88978769 & 1.28540046 \\
\hline $\mathrm{H}$ & 0.43644194 & -2.89047639 & 2.17966785 \\
\hline $\mathrm{H}$ & 1.71001899 & -3.77400228 & 1.32213231 \\
\hline $\mathrm{H}$ & 1.70827443 & -2.00431628 & 1.32227229 \\
\hline $\mathrm{N}$ & -2.03868788 & -3.58967877 & 0.00000000 \\
\hline $\mathrm{H}$ & -1.52286193 & -6.01628181 & 0.00000000 \\
\hline $\mathrm{H}$ & 1.53265707 & -5.54680063 & 0.00000000 \\
\hline $\mathrm{C}$ & 0.59554437 & -22.80175462 & 0.00000000 \\
\hline $\mathrm{C}$ & 0.80844063 & -21.37512816 & 0.00000000 \\
\hline $\mathrm{C}$ & -0.19951475 & -20.23472399 & 0.00000000 \\
\hline $\mathrm{C}$ & 0.81102805 & -19.09672800 & 0.00000000 \\
\hline $\mathrm{C}$ & 0.60186183 & -17.66953717 & 0.00000000 \\
\hline
\end{tabular}




\begin{tabular}{|c|c|c|c|}
\hline $\mathrm{N}$ & 2.04361280 & -20.93459710 & 0.00000000 \\
\hline $\mathrm{C}$ & -1.06229830 & -20.23365807 & 1.28548107 \\
\hline $\mathrm{H}$ & -1.70510529 & -21.11762919 & 1.32235571 \\
\hline $\mathrm{H}$ & -1.70273193 & -19.34795972 & 1.32246386 \\
\hline $\mathrm{H}$ & -0.43105196 & -20.23457339 & 2.17961913 \\
\hline $\mathrm{C}$ & -1.06229830 & -20.23365807 & -1.28548107 \\
\hline $\mathrm{H}$ & -0.43105196 & -20.23457339 & -2.17961913 \\
\hline $\mathrm{H}$ & -1.70273193 & -19.34795972 & -1.32246386 \\
\hline $\mathrm{H}$ & -1.70510529 & -21.11762919 & -1.32235571 \\
\hline $\mathrm{N}$ & 2.04512085 & -19.54007873 & 0.00000000 \\
\hline $\mathrm{C}$ & -0.58876678 & -11.88714461 & 0.00000000 \\
\hline $\mathrm{C}$ & -0.79963816 & -13.31403270 & 0.00000000 \\
\hline $\mathrm{C}$ & 0.20911028 & -14.45361294 & 0.00000000 \\
\hline $\mathrm{C}$ & -0.80059003 & -15.59239693 & 0.00000000 \\
\hline $\mathrm{C}$ & -0.59075386 & -17.01943139 & 0.00000000 \\
\hline $\mathrm{N}$ & -2.03446156 & -13.75549003 & 0.00000000 \\
\hline $\mathrm{C}$ & 1.07205235 & -14.45395558 & -1.28534960 \\
\hline $\mathrm{H}$ & 1.71409966 & -13.56938713 & -1.32207700 \\
\hline $\mathrm{H}$ & 1.71329140 & -15.33909608 & -1.32213167 \\
\hline $\mathrm{H}$ & 0.44106385 & -14.45365575 & -2.17967283 \\
\hline $\mathrm{C}$ & 1.07205235 & -14.45395558 & 1.28534960 \\
\hline $\mathrm{H}$ & 0.44106385 & -14.45365575 & 2.17967283 \\
\hline $\mathrm{H}$ & 1.71329140 & -15.33909608 & 1.32213167 \\
\hline $\mathrm{H}$ & 1.71409966 & -13.56938713 & 1.32207700 \\
\hline $\mathrm{N}$ & -2.03508685 & -15.14990611 & 0.00000000 \\
\hline $\mathrm{H}$ & -1.52250837 & -17.57700407 & 0.00000000 \\
\hline $\mathrm{H}$ & 1.53374974 & -17.11212165 & 0.00000000 \\
\hline $\mathrm{H}$ & 1.53578232 & -11.79599277 & 0.00000000 \\
\hline $\mathrm{H}$ & -1.52013672 & -11.32891177 & 0.00000000 \\
\hline $\mathrm{H}$ & -1.52899355 & -22.88786229 & 0.00000000 \\
\hline $\mathrm{H}$ & 1.52576158 & -23.36192658 & 0.00000000 \\
\hline $\mathrm{H}$ & 1.52816435 & -0.23229079 & 0.00000000 \\
\hline $\mathrm{H}$ & -1.52816435 & 0.23229079 & 0.00000000 \\
\hline
\end{tabular}

